NASA Technical Paper 1302

LOAN COPY: RETURN -

AFWL TECHNICAL LIBR

KIRTLAND AFB, N. M

Critical Study of Higher Order

Numerical Methods for Solving

the Boundary-Layer Equations

Stephen F. Wornom

NOVEMBER 1978 
NASA Technical Paper 1302

\section{Critical Study of Higher Order Numerical Methods for Solving the Boundary-Layer Equations}

Stephen F. Wornom

Langley Research Center

Hampton, Virginia

\section{N/Sก \\ National Aeronautics \\ and Space Administration}

Scientific and Technical Information Office 
A fourth-order box method is presented for calculating numerical solutions to parabolic, partial-differential equations in two variables or ordinary differential equations. The method, which is the natural extension of the secondorder box scheme to fourth order, is demonstrated with application to the incompressible, laminar and turbulent, boundary-layer equations. The efficiency of the present method is compared with other two-point and three-point higher order methods, namely, the Keller box scheme with Richardson extrapolation, the method of deferred corrections, a three-point spline method, and a modified finiteelement method. For equivalent accuracy, numerical results show the present method to be more efficient than the other higher order methods for both laminar and turbulent flows.

\section{INTRODUCTION}

Some of the recent effort in the application of numerical methods to the boundary-layer equations has been directed toward finite-difference methods which have truncation errors that are of higher order than the second-order methods in use today. The term higher order refers to the truncation error in the coordinate normal to the body surface. The truncation error in the tangential coordinate is second order in all the methods discussed in this report. The advantages of higher order methods are twofold. First, they can be used to obtain a numerical solution as accurate as a second-order method with less computer time and storage; alternatively, they can be used to produce a significantly more accurate solution with the same amount of computer time and storage as a second-order method.

The higher order methods can be either composite or intrinsic. Composite methods rely on one or more second-order calculations to achieve their higher order accuracy. Richardson extrapolation and the method of deferred corrections, both discussed by Keller (ref. 1), when applied to second-order methods are examples of composite methods. Intrinsic methods solve a single set of governing difference equations which have a truncation error smaller than second order and whose solution does not depend on a previous second-order calculation.

The higher order intrinsic methods proposed for the boundary-layer equations consist of finite-difference schemes which involve two or three grid points. The three-point schemes fall into two classes. The first class consists of procedures which are fourth order for uniform grids. These procedures treat the functional value and certain derivatives as unknowns at three collocation points and can be derived via Taylor series or polynomial interpolation. In this category are the Padé approximation of Kreiss (ref. 2), or the socalled compact scheme (ref. 3), the Mehrstellen formulation (ref. 4), and the formulation of Peters (ref. 5). The second class of three-point schemes consists of methods for variable grids such as the spline collocation methods of 
Rubin and Graves (refs. 6 and 7) and Rubin and Khosla (refs. 8 and 9). Adam (refs. 10 and 11) and Kordulla (private communication) have extended the compact and Mehrstellen schemes, respectively, to variable grids. The two classes of three-point schemes are similar in that the resulting finite-difference equations involve three nodal points but are different in that the first class is restricted to uniform grids whereas the second class is applicable to variable grids. The emphasis in this study is on methods applicable to variable grids.

One disadvantage of higher order intrinsic methods involving three nodal points is that the usual boundary conditions for incompressible flow (i.e., no slip and no injection at the surface and the tangential velocity component approaches the edge value as the boundary layer merges with the mainstream) are no longer sufficient, since the resulting general system of finite-difference equations contains more unknowns than equations. This difficulty is usually finessed by inventing one or more additional boundary conditions at the outer edge of the layer and by applying an additional equation at the surface boundary. The choice of which additional boundary conditions to use is not clear.

Another disadvantage of some higher order intrinsic methods is the complexity of the resulting nonlinear, finite-difference equations and the associated difficulty in solving them efficiently at each column. For example, the spline 4 method of Rubin and Khosla (refs. 8 and 9) would seem to require the solution of a $5 \times 5$ block-tridiagonal matrix to solve the fully coupled, incompressible, boundary-layer equations. A simpler solution scheme, which lags the continuity equation one iteration behind the momentum equation, is reported by Rubin and khosla (ref. 8). Since the equations in this scheme are solved uncoupled, the errors diminish in a linear manner with each iteration. In contrast, the better second-order methods, such as the Davis coupled scheme (ref. 12) and the Keller box scheme (ref. 13), solve the equations fully coupled with Newton linearization, and thus, for laminar flows, quadratic convergence is achieved. Hence, the advantages of some higher order methods, relative to second-order methods, may be reduced or lost entirely in practical engineering calculations because of slow convergence of the nonlinear system.

The main purpose of this report is to present a fourth-order intrinsic box scheme for obtaining numerical solutions to parabolic, partial-differential equations or ordinary differential equations, with application here to the steady, two-dimensional, incompressible, laminar or turbulent, boundary-layer equations. The method has the following features: (1) It results in finitedifference equations that involve only two nodal points and therefore is formally fourth-order accurate on all grids, (2) it results in a $3 \times 3$ matrix of unknowns at each nodal point when the equations are solved in a coupled manner, (3) it utilizes Newton linearization and demonstrates quadratic convergence for laminar flows, and (4) it requires only the standard incompressible boundary conditions given previously. In short, the method is the natural fourth-order extension of the second-order box scheme. It is an example of a general class of high-accuracy, two-point methods examined by white (ref. 14) and discussed briefly by Keller (ref. 15). Keller offers an operation-count analysis that suggests that such methods may be superior to the well-known Keller box scheme (with Richardson extrapolation) to achieve accuracy less than or equal to six order. 
The second purpose of this report is to compare the present fourth-order box scheme with other proposed or existing higher order methods, including a second-order method with Richardson extrapolation, the method of deferred corrections, a three-point spline scheme, and a modified finite-element scheme.

\section{SYMBOLS}

$A_{n}, B_{n} \quad 3 \times 3$ matrices defined by equation (27)

$a_{i j} \quad i, j$ th element of $A_{n}$

$b_{i j} \quad i, j$ th element of $B_{n}$

C constant in equation (37); also $3 \times 3$ matrix given by equation (B2)

$\bar{C} \quad$ constant in equation (38)

$C_{f} \quad$ surface skin-friction coefficient, $\tau_{\mathfrak{w}}^{*} /\left(\frac{1}{2} \rho_{\infty}^{\star} U_{\infty}^{\star 2}\right)$

$c_{0}, c_{1} \quad$ constants in grid-stretch function (see eq. (35))

$\tilde{c}_{1}, \tilde{c}_{2}, \tilde{c}_{3}$ constants in finite-difference expression for $\partial() / \partial \xi$ terms (eq. (A1))

$D_{n} \quad 3 \times 3$ matrix given by equation (B8)

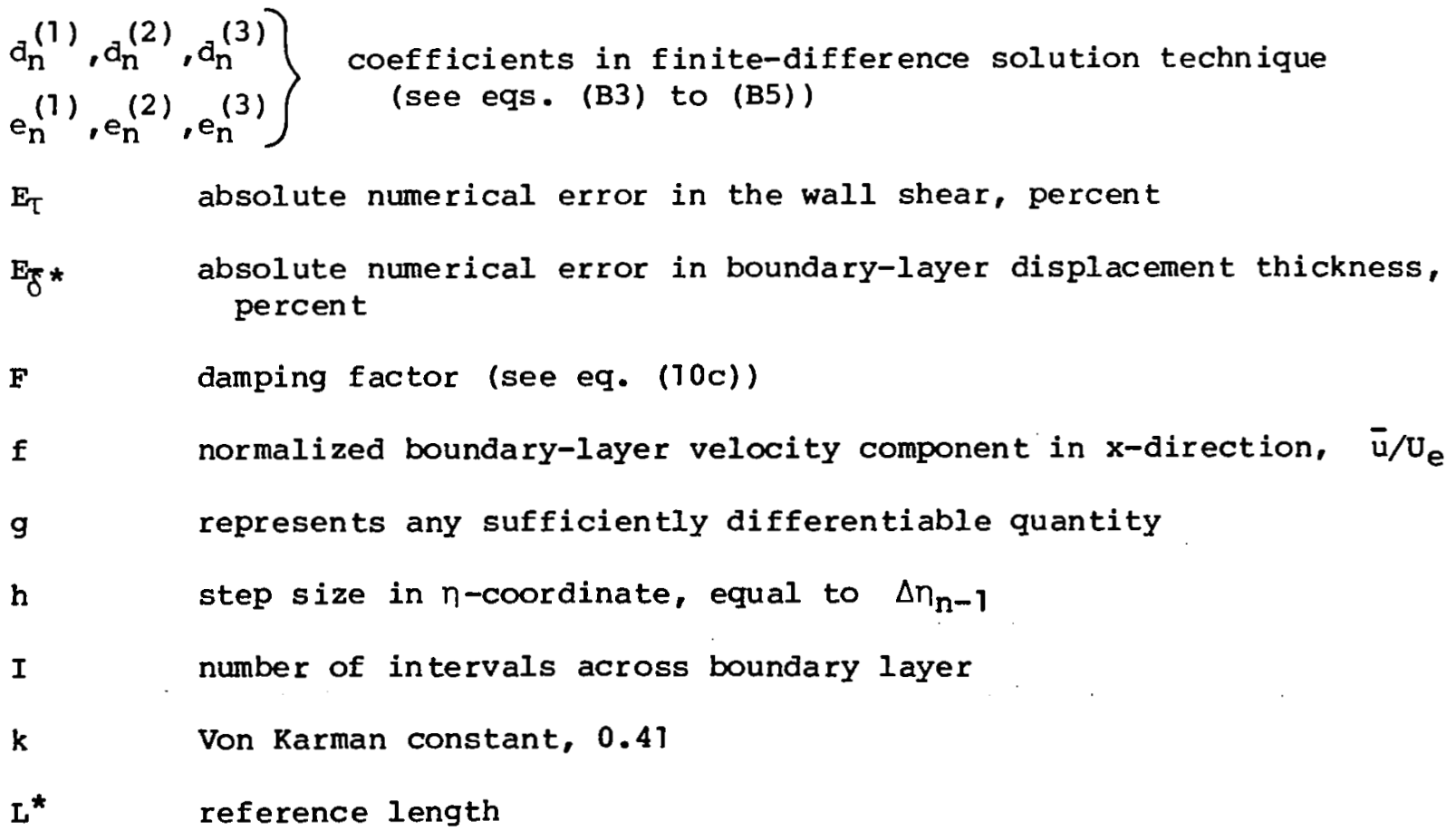


scale length measured in $\mathrm{Y}$-coordinate

$\ell=1+\varepsilon$

N number of grid points across boundary layer

$p_{n}, q_{n}, r_{n}$ components of vector $\vec{w}_{n}$ (see eq. (26))

$R_{n} \quad$ correction term (see eq. (33b))

$R_{L} \quad$ Reynolds number based on $L^{*}, \rho_{\infty}^{*} U_{\infty}^{*} L^{*} \mu_{\infty}^{*}$

$\mathrm{R}_{\mathrm{X}} \quad$ Reynolds number based on $\mathrm{x}^{*}, \rho_{\infty}^{*} \mathrm{U}_{\infty}^{*} \mathrm{x}^{*} \mu_{\infty}^{*}$

s $\quad=\partial \mathrm{f} / \partial n$

T central-processor-unit time

$t \quad$ defined by equation $(10 \mathrm{~b})$

$U^{*} \quad$ inviscid flow velocity in $x$-direction

$\mathrm{U} \quad \mathrm{U} * / \mathrm{U}_{\infty}^{*}$

$\bar{u} * \quad$ time-averaged viscous flow component in $x$-direction

$\bar{u} \quad \bar{u} * / N_{\infty}^{*}$

$\begin{array}{ll}u_{\tau}^{*} & \text { friction velocity, } \sqrt{\mid \tau_{\frac{w}{*}}^{*} / \rho_{\infty}^{*}} \\ \overline{v^{*}} & \text { time-averaged viscous flow component in Y-direction } \\ \bar{v} & =\sqrt{R_{L}} \bar{v}^{*} / \mathrm{O}_{\infty}^{*}\end{array}$

$v \quad$ transformed viscous flow component in $y$-direction (see eq. (7))

$\vec{w}_{n} \quad$ vector defined by equation (26)

$x^{*} \quad$ position coordinate measured along body surface from leading edge or stagnation point

$\mathbf{x}=x^{*} / L^{*}$

Y stretched normal coordinate, $\sqrt{\mathrm{R}_{\mathrm{L}} \mathrm{Y}}$

$y^{*} \quad$ physical distance normal to body surface, $y=0$ at surface

$y=y^{*} / L^{*}$ 


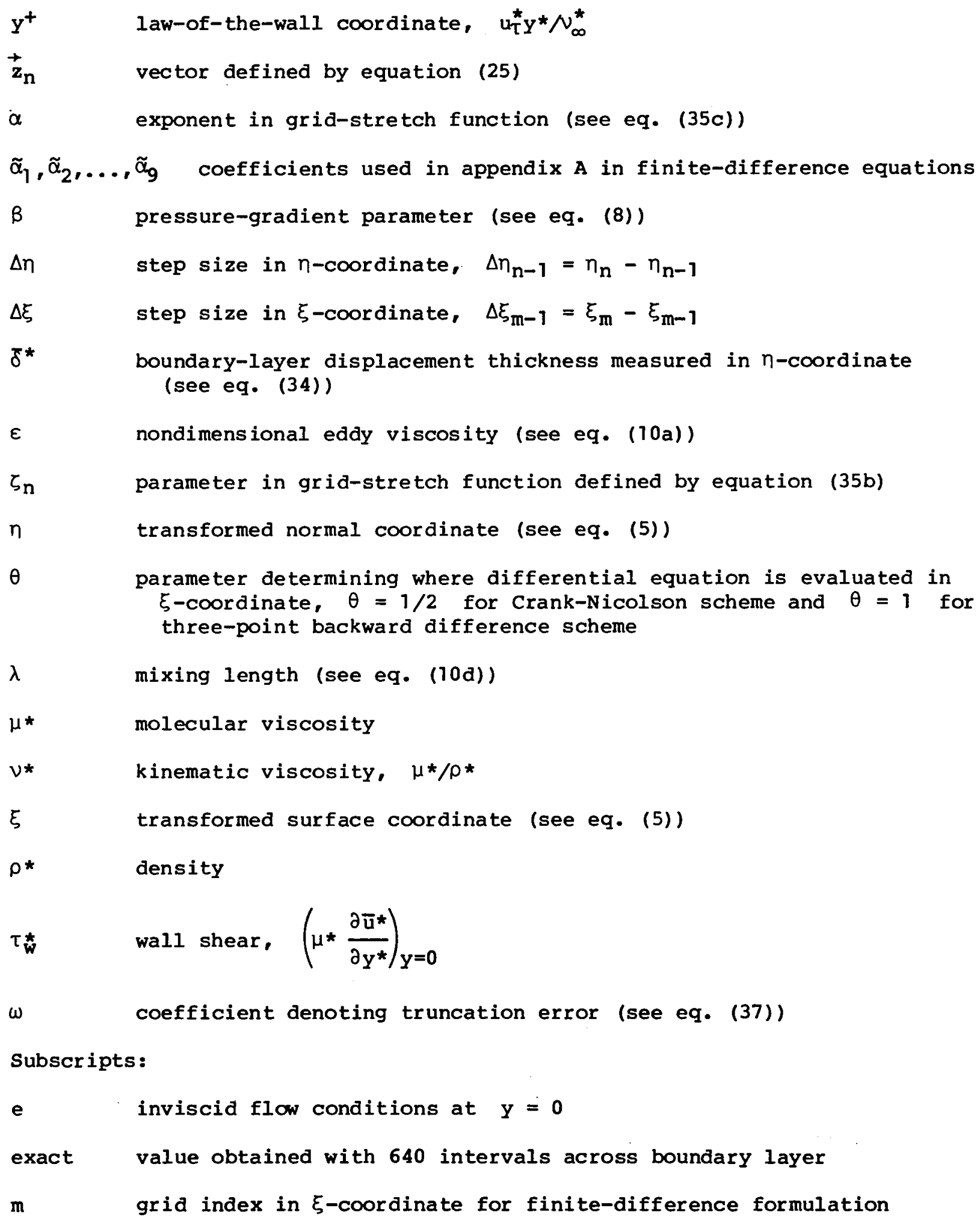




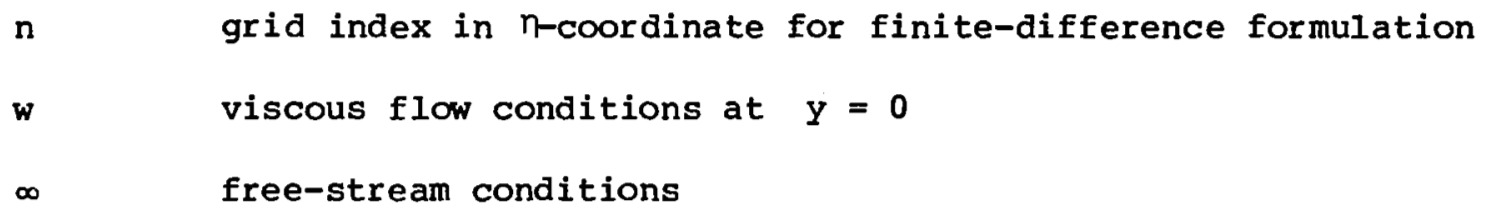

The present method is demonstrated with application to the steady, twodimensional, incompressible, laminar or time-averaged turbulent, boundary-layer equations (ref. 16, p. 545) which are expressed here in Görtler variables (ref. 17): 
Momentum

$$
\frac{\partial}{\partial \eta}\left(\ell \frac{\partial f}{\partial \eta}\right)-v \frac{\partial f}{\partial \eta}-\beta\left(f^{2}-1\right)-2 \xi f \frac{\partial f}{\partial \xi}=0
$$

Continuity

$$
\frac{\partial v}{\partial \eta}+2 \xi \frac{\partial f}{\partial \xi}+f=0
$$

Boundary conditions are

$$
\begin{aligned}
& f=v=0 \text { at } \eta=0 \text { (no slip, no injection) } \\
& f \rightarrow 1 \text { as } \eta \rightarrow \infty
\end{aligned}
$$

Other quantities are given by

$$
\begin{aligned}
& \xi=\int_{0}^{\mathrm{x}} \mathrm{U}_{\mathrm{e}}(\phi) \mathrm{d} \phi \quad \eta=\frac{\mathrm{U}_{\mathrm{e}}(\mathrm{x})}{\sqrt{2 \xi}} \mathrm{Y} \\
& \mathrm{f}=\frac{\overline{\mathrm{u}}}{\mathrm{U}_{\mathrm{e}}} \\
& \mathrm{v}=\frac{\sqrt{2 \xi}}{\mathrm{U}_{\mathrm{e}}} \overline{\mathrm{v}}+\frac{2 \xi \mathrm{f} \partial \mathrm{\partial} / \partial \mathrm{x}}{\mathrm{U}_{\mathrm{e}}} \\
& \beta=\frac{2 \xi}{\mathrm{U}_{\mathrm{e}}} \frac{\mathrm{dU} \mathrm{e}}{\mathrm{d} \xi} \\
& \ell=1+\varepsilon
\end{aligned}
$$

The eddy viscosity $\varepsilon$ is given by reference 18:

$$
\varepsilon=t\left|\frac{\partial f}{\partial \eta}\right|
$$

where

$$
t=\frac{F^{2} \lambda 2 \sqrt{R_{L}} U_{e}^{2}}{\sqrt{2 \xi}}
$$




$$
\begin{array}{ll}
F=1-\exp \left(\frac{-y^{+}}{26}\right) & \\
\frac{\lambda}{l}=0.085 \tanh \left(\frac{k}{0.085} \frac{y}{l}\right) & (k=0.41)
\end{array}
$$

Note that the variables in equations (1) to (10) are all nondimensional.

\section{Method of Solution}

The finite-difference expression around which the present method is formulated is given by

$$
g_{n}-g_{n-1}-\frac{h}{2}\left(g_{n}^{\prime}+g_{n-1}^{\prime}\right)+\frac{h^{2}}{12}\left(g_{n}^{\prime \prime}-g_{n-1}^{\prime \prime}\right)+o\left(h^{5}\right)=0
$$

where $g$ represents any sufficiently differentiable quantity, $h$ is the variable step size in the normal coordinate $n$ (i.e., $\left.h=\eta_{n}-\eta_{n-1}\right)$, and the primes denote differentiation with respect to $\eta$. As mentioned previously, equation (11) is not new. Liniger and willoughby (ref. 19) analyzed the stability of equation (11) as an implicit method for solving initial-value problems for stiff systems of ordinary differential equations. Equation (11) is the fourth-order member of a general class of high-accuracy, two-point equations discussed by white (ref. 14) and Keller (ref. 15). Hirsh (ref. 3) used the expression to formulate boundary conditions for the three-point compact scheme applied to the incompressible driven cavity problem.

A key step in the present procedure, as in the second-order Keller box scheme, is to reformulate the problem in terms of a first-order set of partialdifferential equations. This is done by defining $s \equiv \partial f / \partial \eta$ and rewriting equations (1) and (2):

$$
\begin{aligned}
& \frac{\partial}{\partial \eta}(l s-v f)=2 \xi \frac{\partial f^{2}}{\partial \xi}+(1+\beta) f^{2}-\beta \\
& \frac{\partial f}{\partial \eta}=s \\
& \frac{\partial v}{\partial \eta}=-2 \xi \frac{\partial f}{\partial \xi}-f
\end{aligned}
$$


If a vector $\vec{g}$ is now defined as

$$
\vec{g}=\left\{\begin{array}{c}
l_{s}-v f \\
v
\end{array}\right\}
$$

then $\vec{g}^{\prime}$ is the right-hand sides of equations (12) to (14) and $\vec{g} "$, their derivatives with respect to the n-coordinate:

$$
\vec{g}^{\prime}=\left\{\begin{array}{c}
2 \xi \frac{\partial f^{2}}{\partial \xi}+(1+\beta) f^{2}-\beta \\
s \\
-2 \xi \frac{\partial f}{\partial \xi}-f \\
\vec{g}^{n}=
\end{array}\right\}
$$

where equation ( 1 ) has been used to express $s^{\prime}$ in terms of the dependent variables $f$, s, and $v$ (eq. (22)). Substitution of equations (15) to (23) into equation (11) leads to three, nonlinear, finite-difference equations. The $\partial() / \partial \xi$ terms in these equations are written so that they may be approximated either by a two-point central-difference quotient (the Crank-Nicolson scheme) or by a three-point backward difference method; in either case, the equations are second-order accurate in the $\xi$-coordinate and fourth-order accurate in the $n$-coordinate. These nonlinear equations are then linearized by Newton's method, 
with the exception of the term $t \cdot|s| s$ in equation (22) and the quantities $l$ and $s_{1}$ which are used to compute $\lambda$ and $y^{+}$. Application of Newton's method to linearize the eddy viscosity terms was found to particularly accelerate convergence for the turbulent similarity cases. Newton linearization yields three, linear, finite-difference equations which can be expressed as

$$
A_{n} \vec{z}_{n-1}+B_{n} \vec{z}_{n}=\vec{w}_{n}
$$

where

$$
\begin{aligned}
& \vec{z}_{n}=\left(\begin{array}{cc}
i & s_{m}, n, f_{m, n}^{i}, v_{m, n}^{i}
\end{array}\right)^{T} \\
& \vec{w}_{n}=\left(p_{n}, q_{n}, r_{n}\right)^{T} \\
& A_{n}=\left[\begin{array}{lll}
a_{11} & a_{12} & a_{13} \\
a_{21} & a_{22} & a_{23} \\
a_{31} & a_{32} & a_{33}
\end{array}\right] \quad B_{n}=\left[\begin{array}{lll}
b_{11} & b_{12} & b_{13} \\
b_{21} & b_{22} & b_{23} \\
b_{31} & b_{32} & b_{33}
\end{array}\right]
\end{aligned}
$$

The superscript $i$ in equation (25) denotes the present iteration value and the subscripts $m$ and $n$ are grid indices in the $\xi$ - and $n$-coordinate, respectively. The matrices $A_{n}$ and $B_{n}$ and the vector $\vec{w}_{n}$ are functions of the dependent variables evaluated at the $i-1$ iteration and/or at the previous $\xi$-stations. Equations (24) must be solved repeatedly until an acceptable level of convergence is obtained.

Although the double subscripted matrix elements $a_{i j}$ and $b_{i j}$ change values with the index $n$, it is suppressed for simplicity. Superscript $i$ and subscript $m$ will also be suppressed for the same reason. Appendix $A$ shows how $A_{n}, B_{n}$, and $\vec{t}_{n}$ are obtained.

The boundary conditions are

$$
\begin{aligned}
& \mathrm{f}_{1}=\mathrm{v}_{1}=0 \quad \text { (no slip, no injection) } \\
& \mathrm{f}_{\mathrm{N}}=1
\end{aligned}
$$

It is notewor thy that, since equations (24) can be applied at the outer boundary, the total number of finite-difference equations exactly balances the total number of unknowns. In contrast, a three-point method formulated in terms of $f, s$, and $v$ could not be applied at the outer boundary and would result in more unknowns than equations; hence the method would require additional boundary conditions and equations applied at the boundaries. 
The solution of the resulting linear system of finite-difference equations (24) has been given before; see Cebeci and Smith (ref. 20). The method used here is given in appendix $B$.

\section{HIGHER ORDER METHODS WITH WHICH B4S IS COMPARED}

A classification of the higher order methods examined in this study is given in table $I$. The methods in the table are described in the following sections.

TABLE I.- CLASSIFICATION OF HIGHER ORDER METHODS

\begin{tabular}{|c|c|c|c|c|}
\hline \multirow[b]{2}{*}{ Method } & \multirow[b]{2}{*}{ Type } & \multirow{2}{*}{$\begin{array}{c}\text { Number of grid } \\
\text { points in } \\
\text { finite-difference } \\
\text { equations }\end{array}$} & \multicolumn{2}{|c|}{ Formal accuracy with - } \\
\hline & & & $\begin{array}{l}\text { Uniform } \\
\text { grids }\end{array}$ & $\begin{array}{l}\text { Variable } \\
\text { grids }\end{array}$ \\
\hline B4S & Intrinsic & 2 & Fourth order & Fourth order \\
\hline RKS1 & Intrinsic & 3 & Fourth order & Third order \\
\hline MFE & Intrinsic & 3 & Fourth order & Third order \\
\hline CKBSRE & Composite & 2 & Fourth order & Fourth order \\
\hline MDC & Composite & 2 & Fourth order & Fourth order \\
\hline
\end{tabular}

Intrinsic Methods

Three-point spline methods.- Two three-point spline methods were examined in this study. Both methods are formally fourth-order accurate for uniform grid spacing and can also be shown to be fourth order for analytical variable grid stretchings. They are the spline 4 method of Rubin and Khosla (ref. 8), which solves the momentum and continuity equations in an uncoupled manner, and the spline $S^{7}(4,0)$ method, which solves the equations coupled. The equations for the $S^{1}(4,0)$ method were given by Rubin and Khosla (ref. 9) without application. However, since this method is easily applied to solve the coupled momentum and continuity equations with dependent variables $f, s=f '$, and $v$ and thus achieves quadratic convergence for laminar flows, the three-point spline method used for comparison here is the $S^{1}(4,0)$ method. Numerical results obtained with the spline 4 the $s^{1}(4,0)$ methods were virtually identical.

Rubin-Khosla spline $S^{1}(4,0)$ (RKS1). - The RKS1 method is shown here applied to the momentum equation in similarity form which is obtained by deleting the $\partial($ ) $/ \partial \xi$ term in equation (1). To apply the spline method to turbulent flow, the momentum equation is written in the following nonconservative form:

$$
\ell f^{n}+\left(l^{\prime}-v\right) f^{\prime}-\beta\left(f^{2}-1\right)=0
$$


In the RKS1 method, the $f^{\prime \prime}$ derivative in the momentum equation is replaced by a spline relation involving the dependent variables $s$ and $f$ at three node points. The resulting finite-difference momentum equation is then coupled with finite-difference expressions for the continuity equation and an auxiliary equation which enforces spline continuity; both are obtained via spline relations.

After linearizing the finite-difference momentum equation by Newton's method with the exceptions for eddy viscosity noted in the B4S, these $3 \times 3$ block-tridiagonal equations are solved simultaneously for $s_{n}, f_{n}$, and $v_{n}$ with boundary conditions,

$$
\begin{aligned}
& f_{1}=v_{1}=0 \quad \text { (no slip, no injection) } \\
& f_{N}=1
\end{aligned}
$$

Since three-point finite-difference equations can be applied only at interior node points, there are three more unknowns than equations. However, $v_{N}$ does not appear in the finite-difference equations written in nonconservative form, so that only two more unknowns than equations remain. The two additional equations needed are obtained by setting $s_{N}=0$ and evaluating $s_{1}$ by applying equation (11) at $n=2$ to the momentum equation (12).

Modified finite-element method (MFE).- The modified finite-element method of Rubin and Khosla (ref. 21) differs from the three-point spline methods in that the equations are solved in conservative form. The momentum finitedifference equation is given by

$$
\begin{aligned}
& g_{n+1}-g_{n-1}-\frac{h}{2}\left[\sigma g_{n+1}^{\prime}+(1+\sigma) g_{n}^{\prime}+g_{n-1}^{\prime}\right] \\
& +\frac{h^{2}}{12}\left[\sigma^{2} g_{n+1}^{\prime \prime}-\left(\sigma^{2}-1\right) g_{n}^{\prime \prime}-g_{n-1}^{\prime \prime}\right]=0
\end{aligned}
$$

where $\sigma=\Delta n_{n} / \Delta n_{n-1}$ and $g_{n}, g_{n}^{\prime}$, and $g_{n}^{\prime \prime}$ are given by equations (15), (18), and (21). The continuity equation is the same as the B4S finitedifference expression (eq. (11)). These equations are coupled with an auxiliary equation which enforces spline continuity.

After linearizing the momentum equation with the exceptions for eddy viscosity noted in the B4S, these $3 \times 3$ block-tridiagonal equations are rewritten with dependent variables $f, v$, and $M=f "$ subject to the boundary conditions given in equations $(30)$.

The coupled system of equations for $f_{n}, v_{n}$, and $M_{n}$ written in this form is unstable when numerical solutions are attempted. However, a stable system can be obtained by eliminating $v_{n+1}$ and $v_{n-1}$ from the momentum difference equation via the continuity equation. A computer code listing which supplied details of this method was provided courtesy of Rubin and Khosla. 
Since $v_{N}$ no longer appears in the difference equations, only two additional equations are required. The momentum equation is used to show that $M_{1}=-\beta$. A spline relation relating $s_{N}$ to $M_{N}$ and $f_{N}$ was also used (eq. (22a) in ref. 21 ).

\section{Composite Methods}

Two composite methods, or methods which rely on one or more second-order calculations to achieve their higher order accuracy, were studied. They are Richardson extrapolation and the method of deferred corrections applied to a second-order method; both are discussed by Keller (ref. 1). The second-order method used in the composite method was chosen from among the Keller box scheme (KBS), the conservative Keller box scheme (CKBS), and the Davis coupled scheme (DCS). The CKBS was chosen because it provides more accurate results than the DCS on a coarse grid and is more efficient than the kBS for laminar flows. The three second-order methods are compared in detail in the section "Selection of Second-Order Method."

Conservative Keller box scheme with Richardson extrapolation (CKBSRE) .Application of Richardson extrapolation to obtain higher order accuracy using the second-order Keller box scheme for the boundary-layer equations has been reported by Keller and Cebeci (ref. 22). Richardson extrapolation is applied here to the second-order conservative Keller box scheme (CKBS), which is the second-order method obtained when the higher order terms are deleted from the B4S. The extrapolated solution $f_{n}^{e}$ for a grid with $I$ intervals is obtained by the following relation:

$$
f_{n}^{e}(I)=f_{2 n-1}(2 I)+\frac{1}{3}\left[f_{2 n-1}(2 I)-f_{n}(I)\right]
$$

where $f_{n}(I)$ is the solution on a grid with $I$ intervals and $f_{n}(2 I)$ is the solution on a grid with $2 I$ intervals. Identical extrapolations are used for the dependent variables $s_{n}$ and $v_{n}$ as well as for the transformed displacement thickness $\bar{\delta}^{\star}$. Keller (ref. 1) has shown that for second-order methods, a single Richardson extrapolation should result in a solution with accuracy $O\left(I^{-4}\right)$.

Method of deferred corrections (MDC).- The method of deferred corrections is combined with the CKBS by applying equation (11) in the form

$$
g_{n}-g_{n-1}-\frac{h}{2}\left(g_{n}^{\prime}+g_{n-1}^{\prime}\right)=R_{n}
$$

where

$$
R_{n}=-\frac{h^{2}}{12}\left(g_{n}^{n}-g_{n-1}^{\prime \prime}\right)
$$


To obtain a solution with the MDC, a converged second-order solution is first obtained with $R_{n}=0$. The solution thus obtained is used to evaluate $R_{n}$ using the functions for $g^{n}$ given in equations (21) to (23). A second converged solution is then obtained for equation (33a) with $R_{n}$ fixed at the value obtained from the first calculation.

This formulation of the method of deferred corrections requires only one deferred correction to make the truncation error formally fourth order which is consistent with the B4S. When applying the method of deferred corrections, there are basically two approaches in approximating the higher order corrections; both are discussed by Keller (ref. 1).

The usual approach is to approximate the derivatives $g_{n}^{\prime \prime}$ and $g_{n-1}^{\prime \prime}$ by three-point central differences; thus the values of $g_{n+1}, g_{n}, g_{n-1}$, and $g_{n-2}$ are required to evaluate $R_{n}$. For uniform grids, the truncation error of this approach is formally fourth order for one deferred correction, whereas for a variable grid, the formal truncation error is increased to third order. This approach also leads to difficulties at the boundaries, where $9_{\mathrm{N}+1}$ and $g_{-1}$ are required to compute $R_{N}$ and $R_{2}$. Pereyra (ref. 23) avoided this difficulty by using one-sided differences at the boundaries for the correction terms. Keller discusses a simpler, and probably superior, procedure for treating the corrections near the boundaries: Extend the second-order numerical solution beyond the boundaries to generate values for $g_{N+1}, g_{N+2}$, . . and $g_{-1}, g_{-2}$, . ., as needed for central differences near or at the boundaries.

An alternative approach for approximating the higher order correction terms, and the one adopted here, is to differentiate the governing equations to obtain $g_{n}^{\prime \prime}$ as a function of the dependent variables $f_{n}, s_{n}$, and $v_{n}$. While this procedure loses its attractiveness when more than one deferred correction is made (i.e., accuracy greater than $O\left(h^{4}\right)$ is desired), it appears to be more efficient for one correction because no modifications are needed at the boundaries and it also has the advantage of remaining formally fourth-order accurate for variable grids.

\section{CALCULATION OF DISPLACEMENT THICKNESS}

The transformed displacement thickness,

$$
\bar{\delta}^{*}=\int_{0}^{\infty}(1-f) d \eta
$$

is computed for the higher order intrinsic methods and the MDC by using equation (11) where $g^{\prime}=1-f$ and thus $g^{\prime \prime}=-s$. Equation (11) is integrated

from the solid boundary, where $g_{1}=0$, to the outer boundary, where $g_{N}=\bar{\delta}^{*}$. The displacement thickness for the CKBSRE is obtained in a similar manner with the higher order terms omitted. 
The author carefully programmed each method with the same efficiencies to obtain a fair comparison with respect to computer run times. The method used to solve the $3 \times 3$ block-tridiagonal finite-difference equations was coded with zero elements eliminated. Several model problems were solved with each method. Comparisons between the second-order methods and between the fourth-order methods were made for laminar cases with $\beta=-0.1,0$, and 1 , and comparison between the fourth-order methods was made for similar and nonsimilar turbulent cases with $B=0$ and 0.5 . The same initial profiles were used with each method. The initial profiles are given by $f_{n}=1-e^{-a n_{n}}, \quad s_{n}=a\left(1-f_{n}\right)$, and $v_{n}=-n_{n}$ where $a=0.47,0.47$, and 1.23 corresponding to the three values of $\beta$ for the laminar cases and $a=2.75$ and 3.42 corresponding to the two values of $\beta$ for the turbulent cases.

Since a closed-form solution to the governing equations is not available, the "exact" solution was taken to be the B4S solution with 640 intervals (641 grid points) across the boundary layer, unless otherwise noted.

\section{Selection of Second-Order Method}

In selecting the second-order method to use with Richardson extrapolation and the method of deferred corrections, three second-order methods were studied. They are the Keller box scheme (KBS), the conservative Keller box scheme (CKBS), and the Davis coupled scheme (DCS). The CKBS is the second-order method obtained when the higher order terms are deleted from the B4S. It differs from the KBS (ref. 13) mainly in the form of the momentum finite-difference equation which is written in conservation form while the KBS version is obtained from equation (1) which is only partially conservative. The dependent variables for both methods are $f, s=f^{\prime}$, and $v$. The DCS (ref. 12) uses a three-point variable-grid scheme reported by Blottner (ref. 24) with the linearized finitedifference equations for $f$ and $v$ being solved with a modified tridiagonal scheme. All three methods solve the momentum and continuity equations coupled and are linearized via Newton's method.

As noted by Keller and Cebeci (ref. 22), the purpose of higher order methods is not to acquire more significant digits in the solution but rather to get reasonable answers with relatively few grid points. With this purpose in mind, the second-order method selected was the one which gave the best results with the fewest number of grid points for model problems having favorable as well as adverse pressure gradients.

The second-order methods are compared for laminar-flow problems only. The governing equations for the model problems reduce to ordinary differential equations and are obtained by deleting the $\partial() / \partial \xi$ terms from the governing equations and setting $\ell=1$. The problems were solved with the following numbers of intervals across the boundary layer: $10,16,20,32,40,64,80,128,160$, and 320 . 
The grid size for all calculations varied across the boundary layer. The equation used to generate the grid is given by

$$
\eta_{n}=\frac{\cos _{n}}{\left(1+c_{1} \zeta_{n}\right)^{\alpha}}
$$

where

$$
\zeta_{n}=\frac{n-1}{N-1}
$$

and $c_{1}$ and $\alpha$ are arbitrary constants chosen here so that for $\mathrm{N}=11$, $\Delta n_{1}=0.05$, and the remaining points are distributed in a reasonable manner. For the laminar calculations where $\eta_{N}=24.2538, c_{j}=-0.4$, and $\alpha=8.26$. The constant $c_{0}$ is obtained by applying equation (35a) at $n=N$. This type of grid stretching was selected because all coarse grid points are also points on the exact-solution grid $(\mathrm{N}=641)$; thus, no interpolation between node points was required to compute the norms of the $f-$ and $s$-profiles which were examined as a measure of the numerical error present in the finite-difference solutions. The $\mathrm{f}$-norm is defined as

$$
\left\|f-f_{\text {exact }}\right\|=\frac{1}{N-2} \sum_{n=2}^{N-1}\left|f_{n}-f_{n, \text { exact }}\right|
$$

with the s-norm being defined in a similar manner. The numerical errors in the wall shear $E_{\tau}$ and in the transformed boundary-layer displacement thickness $\mathrm{E}_{\delta^{*}}$ were also considered in determining the relative accuracy of the methods.

Figure 1 shows the percentage error in the wall shear versus the number of intervals across the boundary layer, $I=N-1$, for the CKBS, KBS, and DCS for laminar, flat-plate $(\beta=0)$ flow. (The slopes indicated in the figures $(2$ to 1 in fig. 1) are the slopes of the lines on the logarithmic grid.) The figure shows that as the grid is refined, the CKBS is the most accurate when accuracy is measured solely by the percentage error in the wall shear. As the grid is refined, the numerical errors for laminar flow have the following form:

$$
E=C I^{-\omega} \text { as } I \rightarrow \infty
$$

where $\omega=2$ for second-order methods and $\omega=4$ for fourth-order methods, with $E$ being the $f$-norm, s-norm, $E_{\tau}$, or $E_{\delta_{*}}$. The constants $C$ were com-

puted for both $\beta=1$ and $\beta=0$. Typical results show that the ratios of the number of intervals to achieve an equivalent accuracy in the wall shear, $I_{K B S} / I_{D C S}$ and $I_{C K B S} / I_{D C S}$, were 2.3 and 0.5 , respectively, for $\beta=0$ and 1.4 and 0.8 , respectively, for $\beta=1$. 
To compute the relative efficiency of the methods, it was observed that, as the grid was refined, the central-processor-unit time in seconds $T$ could be approximated as

$$
\mathbf{T}=\mathbf{i} \bar{C} \mathbf{I}
$$

where $\bar{C}$ is a constant and $i$ is the number of iterations required for convergence. The efficiency of method $A$ relative to method $B$ is given by

$$
T_{A} / T_{B}=\left(\bar{C}_{A} / \bar{C}_{B}\right)\left(I_{A} / I_{B}\right)=\left(\bar{C}_{A} / \bar{C}_{B}\right)\left(C_{A} / C_{B}\right)^{1 / \omega}
$$

where $I_{A} / I_{B}$ is the ratio of the number of intervals to achieve an equivalent accuracy in one of the errors. Because of the short run times, on the Control Data CYBER 175 computer, special care was taken in determining the constants $\overline{\mathrm{C}}$ with the values used being the average over $6400 / I$ calculations. Thus, for 10 intervals, the computer times were averaged over 640 calculations.

For comparison of the three second-order methods, the convergence criterion required that

$$
|\Delta \mathrm{f}|_{\max } \leqq 10^{-12}
$$

where

$$
|\Delta f|_{\max }=\max _{n}\left|f_{n}^{i}-f_{n}^{i-1}\right|
$$

Since all the second-order methods converged quadratically for laminar flows and thus required an equal number of iterations for the cases where time efficiency was computed, it was not felt that this restrictive convergence criterion penalized any method. Typical results showed that to compute an equivalent accuracy in the wall shear, the relative times $\mathrm{T}_{\mathrm{KBS}} / \mathrm{T}_{\mathrm{DCS}}$ and $\mathrm{T}_{\mathrm{CKBS}} / \mathrm{T}_{\mathrm{DCS}}$ were 2.8 and 0.7 , respectively, for $\beta=0$. Similar results were obtained for $\beta=1$. However, the relative efficiency of the methods varied with the pressure gradient parameter $\beta$ and also with the different numerical errors.

Note from equation (39) that the relative time efficiency depends both on the relative time to solve the difference equations, $\overline{\mathrm{C}}_{\mathrm{KBS}} / \overline{\mathrm{C}}_{\mathrm{DCS}}$, and the relative truncation error $\left(C_{\mathrm{KBS}} / \mathrm{C}_{\mathrm{DCS}}\right)^{1 / \omega}$. As the number of intervals becomes small, figure 1 shows the truncation error to deviate from its asymptotic behavior with the DCS showing the greatest deviation. This is borne out in table II where the f-profile obtained on a coarse grid $(N=11)$ is compared with the exact solution. 
TABLE II.- COMPARISON OF SECOND-ORDER F-PROFILES WITH

EXACT SOLUTION FOR $\beta=0$

[Laminar flow; $N=11]$

\begin{tabular}{|c|c|c|c|c|}
\hline \multirow{2}{*}{$\eta$} & \multicolumn{4}{|c|}{ f-profiles calculated with - } \\
\hline & Exact & CKBS & KBS & $\mathrm{DCS}$ \\
\hline $\begin{array}{l}0 \\
.49975 \times 10^{-1} \\
.14205 \times 10^{0} \\
.30761 \times 10^{0} \\
.60231 \times 10^{0} \\
.11266 \times 10 \\
.20651 \times 10 \\
.37657 \times 10 \\
.69004 \times 10 \\
.12809 \times 10^{2} \\
.24254 \times 10^{2}\end{array}$ & $\begin{array}{l}0 \\
.23468 \times 10^{-1} \\
.66705 \times 10^{-1} \\
.14437 \times 10^{0} \\
.28164 \times 10^{0} \\
.51474 \times 10^{0} \\
.83286 \times 10^{0} \\
.99552 \times 10^{0} \\
.10000 \times 10 \\
.10000 \times 10 \\
.10000 \times 10\end{array}$ & $\begin{array}{l}0 \\
.23426 \times 10^{-1} \\
.66585 \times 10^{-1} \\
.14411 \times 10^{0} \\
.28113 \times 10^{0} \\
.51364 \times 10^{0} \\
.83263 \times 10^{0} \\
.10140 \times 10^{1} \\
.99643 \times 10^{0} \\
.10032 \times 10 \\
.10000 \times 10\end{array}$ & $\begin{array}{l}0 \\
.24727 \times 10^{-1} \\
.70282 \times 10^{-1} \\
.15208 \times 10^{0} \\
.29632 \times 10^{0} \\
.53817 \times 10^{0} \\
.85435 \times 10^{0} \\
.10156 \times 10 \\
.99600 \times 10^{0} \\
.10036 \times 10 \\
.10000 \times 10\end{array}$ & $\begin{array}{l}0 \\
.24432 \times 10^{-1} \\
.69448 \times 10^{-1} \\
.15033 \times 10^{0} \\
.29355 \times 10^{0} \\
.53930 \times 10^{0} \\
.89294 \times 10^{0} \\
.10820 \times 10^{2} \\
.99698 \times 10^{0} \\
.10687 \times 10 \\
.10000 \times 10\end{array}$ \\
\hline $\begin{array}{l}E_{\tau}, \text { percent } . . \\
E_{\delta^{\star}}, \text { percent } . .\end{array}$ & & $\begin{array}{r}0.179 \times 10^{0} \\
.167 \times 10\end{array}$ & $\begin{array}{r}0.537 \times 10 \\
.334 \times 10\end{array}$ & $\begin{array}{l}0.411 \times 10 \\
.674 \times 10^{2}\end{array}$ \\
\hline
\end{tabular}

For a coarse grid, the CKBS is slightly more accurate than the KBS. Note the very large error (67 percent) in the displacement thickness for the DCS. This error appears to result from an approximate 8-percent overshoot in the f-profile as compared with 1.5 percent for the CKBS and the KBS.

The solutions obtained with the three-point DCS on a coarse grid $(\mathrm{N}=11)$ showed a sensitivity to the pressure gradient parameter $\beta$ that was not observed with either the CKBS or the KBS. For $\beta=1$, the f-profile for all three methods showed negligible overshoot with errors in the wall shear and displacement thickness that were comparable. However, for $\beta=-0.1$, the errors in the wall shear and displacement thickness for the DCS were 45 and 185 percent, respectively, apparently because of a 21-percent overshoot in the f-profile. For this same case, the CKBS and the KBS showed negligible overshoot with the error in the wall shear and displacement thickness being 1 percent and 5 percent, respectively, for the CKBS.

On the basis of these laminar results on a coarse grid, the CKBS and the KBS were judged preferable to the DCS for use with Richardson extrapolation and the method of deferred corrections when solutions with only a few grid points are desired. Since the CKBS was found to be more efficient than the KBS for laminar flows, the CKBS was chosen to use with the higher order composite methods.

\section{Higher Order Methods Applied to Laminar Flows}

Figure 2 shows the f-norm versus the number of intervals across the boundary layer for the CKBSRE, MFE, $M D C$, and RKSl compared with the B4S values for laminar, stagnation-point $(B=1)$ flow. 
Solutions for model problems using the CKBSRE were first obtained on a grid with 2I intervals and then on a grid with I intervals. The initial profiles for the I-interval solution were taken from the solution with 2I intervals. The error norms for the CKBSRE are shown plotted versus the $I$ intervals. The starting profiles for the MDC calculation where $R_{n} \neq 0$ were those obtained from the $R_{n}=0$ solution. The exact solution $(I=640)$ used to compute the $f$-norm for the RKSl method was obtained with that method, since it required solution of a slightly different boundary problem $\left(s_{N}=0\right)$.

For laminar flow, figure 2 shows all the higher order calculations with a variable grid to be fourth-order accurate as the grid size is refined. The $\mathrm{f}$-norm for the CKBS is shown for comparison. As the grid is refined, the error norms approach the form given in equation (37) with $\omega=4$. Numerical values for the constants $C$ for this case and the laminar, flat-plate $(B=0)$ case were computed.

To determine the relative time efficiency of the higher order methods to achieve equivalent accuracy, the central-processor-unit time in seconds was expressed as in equation (38). The time efficiency was obtained in the same manner as for the second-order methods (eq. (39)).

In a previous paper (ref. 25), the convergence criterion of $|\Delta \mathrm{f}|_{\max } \leqq 10^{-12}$ was also applied to the higher order methods when computing the relative time efficiency. Further studies have shown that this restrictive criterion does in fact penalize the RKS1, MDC, and CKBSRE relative to the B4S.

To obtain a fair comparison, it is necessary only to require that the convergence error be less than the truncation error. This criterion was applied by noting the minimum number of iterations required for the error to plot as shown for $I \leqq 128$ in figure 2. Results for $B=0$ are shown in table III.

TABLE III.- MINIMUM NUMBER OF ITERATIONS REQUIRED

FOR CONVERGENCE FOR $\beta=0$

[ Laminar flow; I $\leqq 128$ ]

\begin{tabular}{|l|c|}
\hline Method & Number of iterations \\
\hline B4S & 5 \\
RKS 1 & 4 \\
MFE & 5 \\
MDC & $\mathrm{a}_{4}+\mathrm{T}$ \\
CKBSRE & $\mathrm{b}_{5}+2$ \\
\hline
\end{tabular}

a The MDC required four iterations to obtain

the $R_{n}=0$ solution and one iteration with the correction term added.

$b_{\text {The CKBSRE required five iterations on the }}$ fine grid and two iterations on the coarse grid. 
TABLE IV. - RELATIVE EFFICIENCY OF HIGHER ORDER METHODS FOR $\beta=0$

[Laminar flow]

\begin{tabular}{|l|c|c|c|}
\hline \multirow{2}{*}{ Relative times } & \multicolumn{3}{|c|}{ For equivalent accuracy in - } \\
\cline { 2 - 4 } & Wall shear & $\bar{\delta}^{*}$ & f-norm \\
\hline $\mathrm{T}_{\mathrm{MDC}} / \mathrm{T}_{\mathrm{B} 4 \mathrm{~S}}$ & 2.3 & 0.9 & 1.4 \\
$\mathrm{~T}_{\mathrm{CKBSRE}} / \mathrm{T}_{\mathrm{B} 4 \mathrm{~S}}$ & 2.3 & 1.1 & 2.0 \\
$\mathrm{~T}_{\mathrm{MFE}} / \mathrm{T}_{\mathrm{B} 4 \mathrm{~S}}$ & 2.7 & 1.4 & 1.9 \\
$\mathrm{~T}_{\mathrm{RKS1}} / \mathrm{T}_{\mathrm{B} 4 \mathrm{~S}}$ & 3.6 & 1.8 & 2.0 \\
\hline
\end{tabular}

Table IV, which shows the relative time efficiency for this case indicates that the B4S is the most efficient higher order method for laminar flows as the grid is refined. Similar results were obtained for $B=1$.

As the number of grid points is reduced, the truncation errors deviate from their asymptotic behavior. Figure 2 shows the MDC and the RKSl to deviate the greatest for the coarse grid, $N=11$. Tables $V$ to VII show the f-profiles obtained on this coarse grid along with the exact solution for laminar flows with favorable as well as adverse pressure gradients $(\beta=1,0$, and -0.1$)$.

TABLE V.- COMPARISON OF HIGHER ORDER F-PROFILES WITH EXACT SOLUTION FOR $B=1$

[Laminar flow; $N=11$ ]

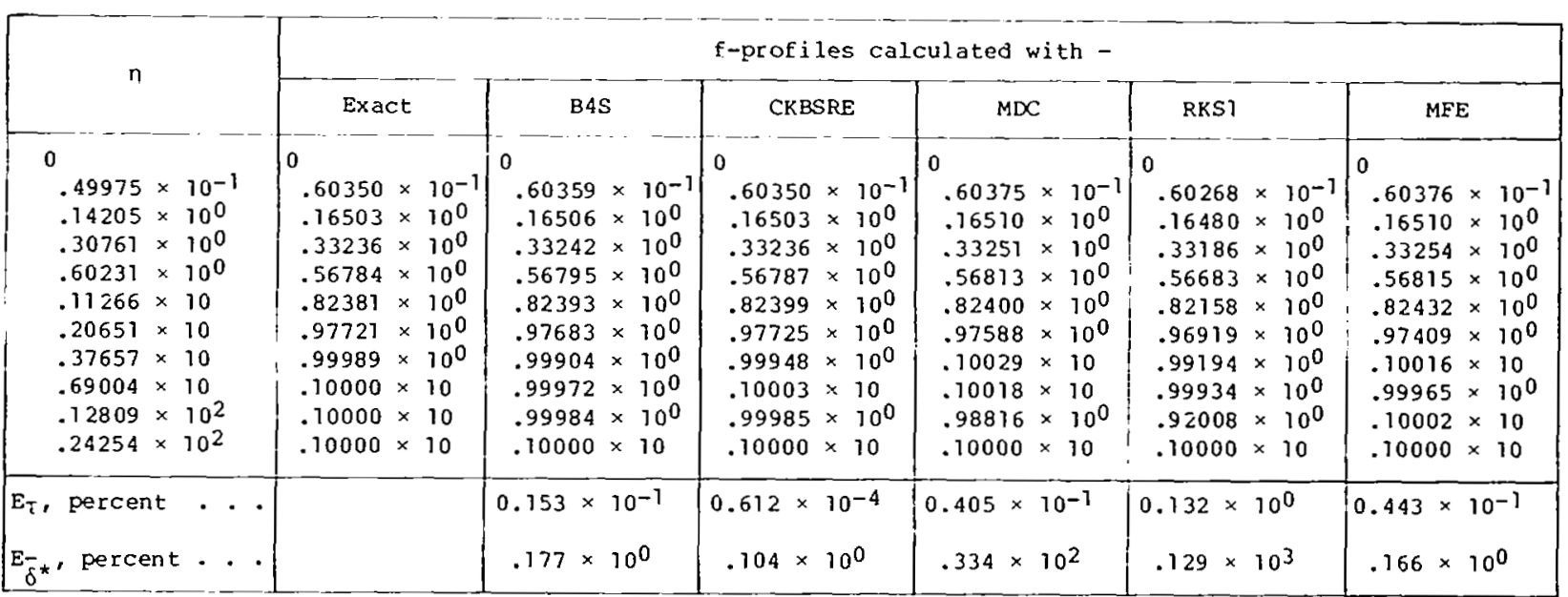


TABLE VI.- COMPARISON OF HIGHER ORDER f-PROFILES WITH EXACT SOLUTION FOR $B=0$

[Laminar flow; $N=11]$

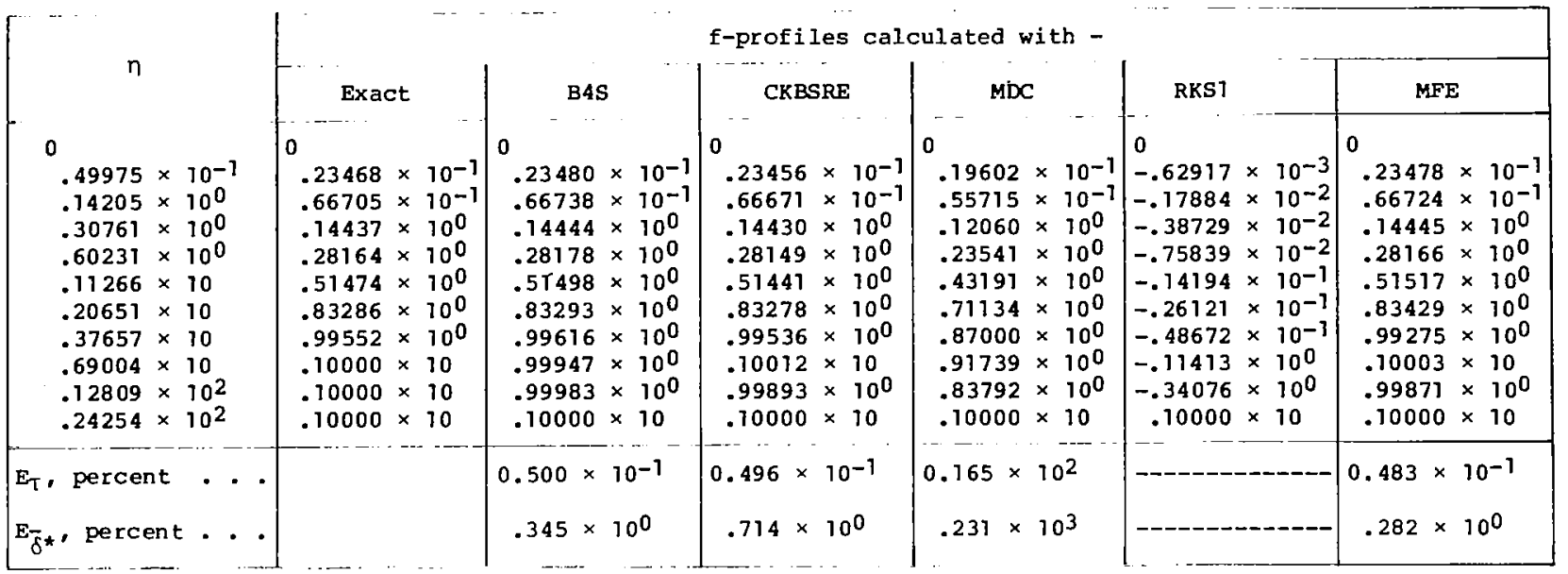

TABLE VII.- COMPARISON OF HIGHER ORDER F-PROFILES WITH EXACT SOLUTION FOR $\beta=-0.1$

[Laminar flow; $N=11$ ]

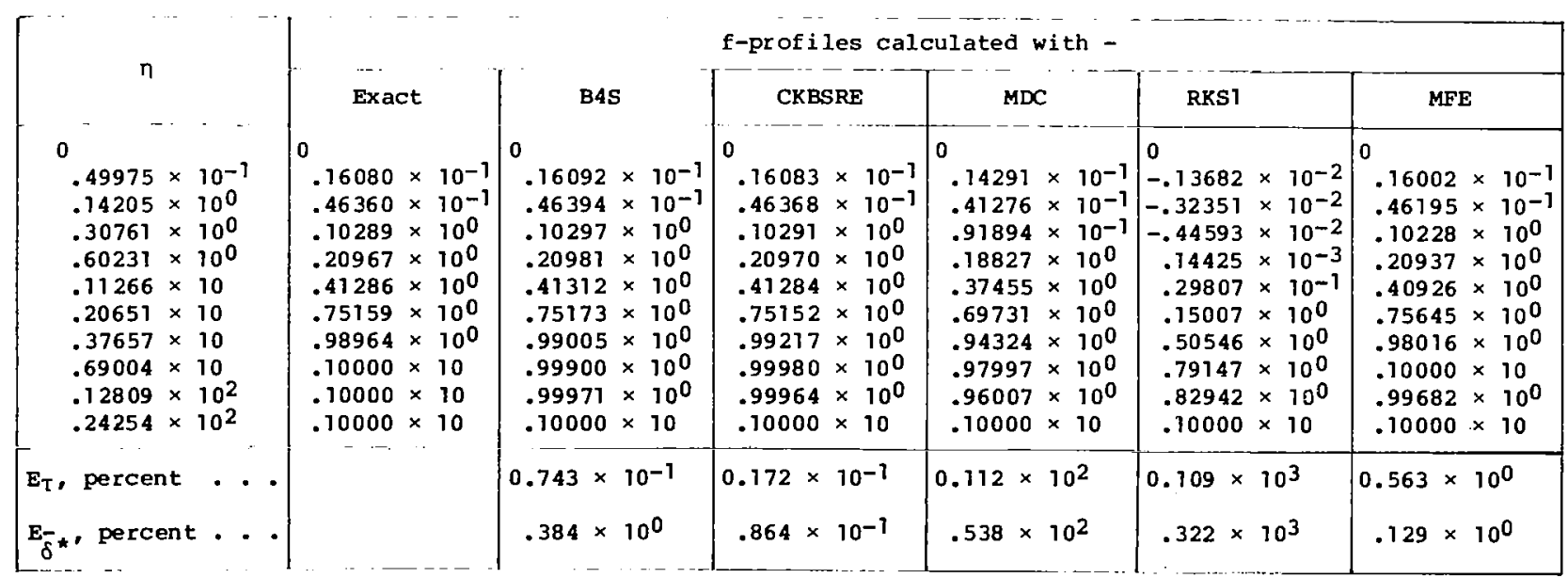


When both displacement thickness and wall shear are considered, table $\mathrm{V}$ $(B=1)$ shows the accuracy of the CKBSRE, MFE, and B4S to be about the same, while the MDC and RKS1 both show significant errors in the displacement thickness. Note that the MDC and the RKS1 show large oscillations in the f-profile. Table VI $(\beta=0)$ shows similar results for the MDC, while the RKS1 method gives a nonphysical solution. Results for an adverse pressure gradient $(B=-0.1)$ are shown in table VII where behavior similar to the other cases $(\beta=1$ and $\beta=0)$ is observed.

\section{Application of Higher Order Methods to Turbulent Flow}

Similar calculations.- Figures 3 and 4 show the percentage error in the wall shear versus the number of intervals across the boundary layer for calculations of a model turbulent problem. The governing equations for these cases are obtained by deleting the $\partial($ ) $/ \partial \xi$ terms from the governing equations. The Reynolds number $R_{L}=1.88 \times 10^{6}, \xi=1$, and $U_{e}=1$, with the outer boundary located at $n_{N}=60$. The constants in the grid-stretch equation (35) are $c_{1}=0.05$ and $\alpha=-109$ and were selected so that for $\mathbf{N}=11, \Delta \eta_{1}=0.05$ and the other points were stretched to $\eta_{N}=60$ in a reasonable manner. The

scale length $\bar{l}=24.5$ for $\beta=0$ and $\bar{l}=15.8$ for $\beta=0.5$. The convergence criterion for these calculations is given by equation (40a). To achieve this convergence, the MDC required multiple iterations with the correction term added.

For $\beta=0$, figure 3 shows the B4S, MFE, and the CKBSRE to be significantly more accurate for the same grid than the MDC and the RKS1, both of which are less accurate than the second-order CKBS for $I<128$. The converged solutions shown for the RKSl were obtained with underrelaxation (relaxation factor $r=0.5$ ). An exact solution (i.e., using 641 grid points) with RKS could not be obtained, and thus, the numerical errors were computed using the exact solution obtained with the B4S.

For $B=0.5$, figure 4 shows the B4S, MFE, and CKBSRE again to be more accurate than the MDC. Converged solutions using RKS1 could not be obtained for the grid sizes shown even with underrelaxation factors $r$ as small as 0.5. However, an exact solution with RKS1 was obtained ( $r=0.7$ ). The exact wall shear values computed with B4S and RKSI agreed to six decimal places.

Because of the roughness of the error curves shown in figures 3 and 4 , it was not feasible to make time efficiency estimates similar to those for laminar flows. The reason for this error scatter in the turbulent cases is not known. However, similar behavior is evident in a plot given by Blottner (ref. 24). The derivative $f^{\prime \prime}$ was observed to be smooth for this case and all other calculations with the B4S.

Nonsimilar calculations.- To determine the relative efficiency of higher order methods for nonsimilar turbulent calculations, the B4S, CRBSRE, MFE, and CKBS were applied to compute the incompressible turbulent boundary layer on a flat plate. The $\xi$-grid is given by $\xi=0,0.00086,0.0043,0.0086,0.043$, $0.17,0.26,0.34,0.51,0.68,0.86,1.03,1.28,1.50,1.71$, and 1.88 . The 
reference Reynolds number $R_{\mathrm{L}}=1 \times 10^{6}$ giving a local Reynolds number $R_{X}=1.88 \times 10^{6}$ at $\xi=1.88$. The $n$-grid was given by equation (35) with $\eta_{\mathbf{N}}=24.2538$. The constants $c_{1}$ and $\alpha$ in the grid-stretch equation were -0.4 and 8.26 , respectively.

Second-order solutions to this problem have been reported by Keller and Cebeci (ref. 22), who also presented higher order results using Richardson extrapolation $(\Delta \eta \rightarrow 0)$, and Blottner (ref. 24). Both of these calculations were made using a two-layer eddy viscosity formulation and a grid-point distribution such that $\Delta n_{n} / \Delta n_{n-1}=$ constant. The grid-point distribution used here closely resembles that used in those previous calculations, that is, $\Delta \eta_{1}=0.05$ for $\mathrm{N}=11$, while a single-layer eddy viscosity model is used for the present solutions.

Figure 5 shows the percentage error in the wall shear versus the number of intervals across the boundary layer at $\xi=1.88$. At $\xi=0.00086$, the CrankNicolson scheme was used to approximate the $\partial(1 / \partial \xi$ terms, while at all other

stations the three-point backward difference scheme was used. The value of $\bar{l}$ was 15.8. Transition was at $\xi=0.00086$.

The exact solution was obtained with 640 intervals across the boundary layer with the $\xi$-grid given previously. Richardson extrapolation for $\Delta \xi \rightarrow 0$ was not made and, as such, the term "exact" means only as the $\Delta n$ grid is refined. The convergence criterion used to obtain the results shown in figure 5 is given by equation $(40 \mathrm{a})$. The only exceptions are for the MFE and B4S where fully converged solutions at the downstream stations could not be reached for $I \leqq 20$ for the MFE and $I \leqq 10$ for B4S.

For laminar calculations, the error norms are smooth and vary according to equation (37) for $\omega=4$. Thus, comparisons for relative time efficiency of the higher order methods can be computed using equation (39) and the results, shown in table IV, apply over a wide range of intervals ( $<128)$. However, for turbulent calculations the error norms are not well behaved and, thus the relative efficiency cannot be computed over a range of intervals as for the laminar calculations.

For nonsimilar turbulent calculations, the efficiencies of the CKBSRE, MFE, and CKBS relative to the B4S were computed for a specific accuracy at a specific point, that is, for a 0.1 -percent numerical error in the wall shear at $\xi=1.88$. The convergence criterion for these comparisons required that the

absolute change in $\bar{\delta}^{\star}$ between consecutive iterations be less than 1 percent. Shown in table VIII are the results where, to obtain this numerical accuracy, $I=12$ for both the B4S and CKBSRE and $I=20$ and 70 for the MFE and CKBS, respectively. Both the MFE and B4S converged without trouble to this criterion, requiring three iterations per streamwise station. For this example, the MFE is a factor of 2 less efficient than the B4S. Note that the second-order CKBS is less efficient than the B4S by a factor of 4.8 . 
TABLE VIII.- RELATIVE EFFICIENCY OF HIGHER ORDER METHODS FOR

NONSIMILAR TURBULENT CALCULATIONS

\begin{tabular}{|l|c|c|}
\hline Method & Number of intervals & Relative efficiency $^{a}$ \\
\hline B4S & 12 & 1.0 \\
MFE & 20 & 2.0 \\
CKBSRE & 12 and 24 & 2.5 \\
CKBS & 70 & 4.8 \\
\hline
\end{tabular}

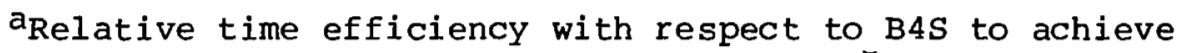
0.1 -percent numerical error in wall shear at $\xi=1.88$, $R_{X}=1.88 \times 10^{6}$.

\section{CONCLUSIONS}

A fourth-order box method for calculating numerical solutions to parabolic, partial-differential equations in two variables or ordinary differential equations has been presented. The method, which is the natural extension of the second-order box scheme to fourth order, has been demonstrated with application to the incompressible, laminar and turbulent, boundary-layer equations. This method has been compared with other two-point and three-point higher order methods on variable grids for laminar and turbulent, incompressible flows. On the basis of these numerical results the following conclusions were reached:

(1) For laminar calculations, the present fourth-order box scheme (B4S), the conservative Keller box scheme with Richardson extrapolation (CKBSRE), the method of deferred corrections (MDC), the modified finite-element method (MFE), and a three-point spline method are fourth-order accurate with variable grids.

(2) For laminar calculations on coarse grids, the B4S, MFE, and CKBSRE give greater accuracy than the MDC and the three-point spline method.

(3) For laminar calculations, the $B 4 S$ is the most efficient higher order method.

(4) For turbulent calculations, the B4S, MFE, and CKBSRE are significantly more accurate than the MDC or the three-point nonconservative spline method.

(5) For a nonsimilar turbulent calculation, the B4S showed a factor of 4.8 reduction in computer time to achieve the same accuracy as the second-order conservative Keller box scheme, a factor of 2 reduction compared with the MFE, and a factor of 2.5 reduction compared with the CKBSRE. 
(6) For practical two-dimensional, incompressible, turbulent boundarylayer calculations (i.e., numerical errors of approximately 0.1 percent), the B4S is the most efficient higher order method.

Langley Research Center

National Aeronautics and Space Administration

Hampton, VA 23665

September 14,1978 
In this appendix, it is shown how to obtain the elements $a_{i j}$ and $b_{i j}$ of the matrices $A_{n}$ and $B_{n}$ and the components $p_{n}, q_{n}$, and $r_{n}$ of the vector $\hbar_{n}$, which appear in equations (24). Equations (24) are the matrix equations which result when equations (15) to (23) are substituted into the finitedifference expression (eq. (11)) and these finite-difference equations are linearized.

The $\partial() / \partial \xi$ terms in equations (18) and (20) to (23) are written in finite-difference form as

$$
\left.\frac{\partial()}{\partial \xi}\right|_{m-(1-\theta), n}=\tilde{c}_{1}()_{m, n}-\tilde{c}_{2}()_{m-1, n}+\tilde{c}_{3}()_{m-2, n}
$$

where $m, n$ are the grid indices in the $\xi, n$ coordinates. The $\partial(1 / \partial \xi$ terms are written so that they may be approximated either by the Crank-Nicolson scheme $\theta=1 / 2)$ where

$$
\tilde{c}_{1}=\tilde{c}_{2}=\frac{1}{\Delta \xi_{m-1}} \quad \tilde{c}_{3}=0
$$

or by a three-point backward-difference scheme $(\theta=1)$ where

$$
\begin{aligned}
& \tilde{c}_{1}=\frac{3}{(1+\gamma) \Delta \xi_{m-2}} \\
& \tilde{c}_{2}=\frac{2}{\Delta \xi_{m-2}} \\
& \tilde{c}_{3}=\frac{2 \gamma-1}{(1+\gamma) \Delta \xi_{m-2}}
\end{aligned}
$$

with

$$
\begin{aligned}
& \gamma=\frac{\Delta \xi_{m-1}}{\Delta \xi_{m-2}} \\
& \Delta \xi_{m-1}=\xi_{m}-\xi_{m-1}
\end{aligned}
$$




\section{APPENDIX A}

The similarity form of the difference equations is obtained by setting $\theta=1$ with $\tilde{c}_{1}=\tilde{c}_{2}=\tilde{c}_{3}=0$.

\section{Momentum Finite-Difference Equation}

Equation (15) is written as

$$
g_{n}=\theta\left(l_{m, n} s_{m, n}-v_{m, n} f_{m, n}\right)^{i}+(1-\theta)\left(l_{m-1, n} s_{m-1, n}-v_{m-1, n} f_{m-1, n}\right)
$$

where $i$ denotes the present iteration. Nonlinear products such as $\left(v_{m}, f_{m, n}\right)^{i}$ are linearized by Newton's method which can be shown to result in

$$
\left(v_{m, n} f_{m, n}\right)^{i}=v_{m, n}^{i-1} f_{m, n}^{i}+v_{m, n}^{i} f_{m, n}^{i-1}-\left(v_{m, n} f_{m, n}\right)^{i-1}
$$

The linearized form of equation (A8) becomes

$$
\begin{aligned}
g_{n}= & \theta\left[\left(2 l_{m, n}^{i-1}-1\right) s_{m, n}^{i}-\left(\ell_{m, n}^{i-1}-1\right) s_{m, n}^{i-1}-v_{m, n}^{i-1} f_{m, n}^{i}-v_{m, n}^{i} f_{m, n}^{i-1}+\left(v_{m, n} f_{m, n}\right)^{i-1}\right] \\
& +(1-\theta)\left(\ell_{m-1, n} s_{m-1, n}-v_{m-1, n} f_{m-1, n}\right)
\end{aligned}
$$

The values of $\xi$ and $\beta$ are defined as

$$
\begin{aligned}
& \xi=\theta \xi_{m}+(1-\theta) \xi_{m-1} \\
& \beta=\theta \beta_{m}+(1-\theta) \beta_{m-1}
\end{aligned}
$$

Equation (18) for $g_{n}^{\prime}$ becomes

$$
\begin{aligned}
g_{n}^{\prime}= & 2 \xi\left[\tilde{c}_{1}\left(f_{m, n}^{2}\right)^{i}-\tilde{c}_{2} f_{m-1, n}^{2}+\tilde{c}_{3} f_{m-2, n}^{2}\right]+(1+\beta)\left[\theta\left(f_{m, n}^{2}\right)^{i}\right. \\
& \left.+(1-\theta) f_{m-1, n}^{2}\right]-\beta
\end{aligned}
$$

Now define

$$
\tilde{\alpha}_{1}=2 \xi \tilde{c}_{1}+(1+\beta) \theta
$$




$$
\begin{aligned}
& \tilde{\alpha}_{2}=-2 \xi_{\tilde{c}_{2}}+(1+\beta)(1-\theta) \\
& \tilde{\alpha}_{3}=2 \xi_{\tilde{c}_{3}}
\end{aligned}
$$

The nonlinear expressions for $g_{n}^{\prime}$ and $g_{n}^{\prime \prime}$ become

$$
g_{n}^{\prime}=\tilde{\alpha}_{1}\left(f_{m, n}^{2}\right)^{i}+\tilde{\alpha}_{2} f_{m-1, n}^{2}+\tilde{\alpha}_{3} f_{m-2, n}^{2}-\beta
$$

and

$$
g_{n}^{\prime \prime}=2\left[\tilde{\alpha}_{1}\left(f_{m, n} s_{m, n}\right)^{i}+\tilde{\alpha}_{2} f_{m-1, n} s_{m-1, n}+\tilde{\alpha}_{3} f_{m-2, n} s_{m-2, n}\right]
$$

After linearizing equations (A17) and (A18) via Newton's method, the linearized expressions for $g_{n}, g_{n}^{\prime}$, and $g_{n}^{\prime \prime}$ are substituted into equation (11), and the resulting equation is rewritten in the following form:

$a_{11} s_{m, n-1}^{i}+b_{11} s_{m, n}^{i}+a_{12} f_{m, n-1}^{i}+b_{12} f_{m, n}^{i}+a_{13} v_{m, n-1}^{i}+b_{13} v_{m, n}^{i}=p_{n}$

where

$$
\begin{aligned}
& a_{11}=-\theta\left(2 l_{m, n-1}^{i-1}-1\right)-\frac{h^{2}}{6} \tilde{\alpha}_{1} f_{m, n-1}^{i-1} \\
& b_{11}=\theta\left(2 l_{m, n}^{i-1}-1\right)+\frac{h^{2}}{6} \tilde{\alpha}_{1} f_{m, n}^{i-1} \\
& a_{12}=\theta v_{m, n-1}^{i-1}-h \tilde{\alpha}_{1} f_{m, n-1}^{i-1}-\frac{h^{2}}{6} \tilde{\alpha}_{1} s_{m, n-1}^{i-1} \\
& b_{12}=-\theta v_{m, n}^{i-1}-h \tilde{\alpha}_{1} f_{m, n}^{i-1}+\frac{h^{2}}{6} \tilde{\alpha}_{1} s_{m, n}^{i-1} \\
& a_{13}=\theta f_{m, n-1}^{i-1} \\
& b_{13}=-\theta f_{m, n}^{i-1}
\end{aligned}
$$




\section{APPENDIX A}

$$
\begin{aligned}
p_{n}= & \theta\left[\left(l_{m, n}-1\right) s_{m, n}-v_{m, n} f_{m, n}-\left(l_{m, n-1}-1\right) s_{m, n-1}+v_{m, n-1} f_{m, n-1}\right]^{i-1} \\
& -\tilde{\alpha}_{1} \frac{h-1}{2}\left[\left(f_{m, n}^{2}\right)^{i-1}+\left(f_{m, n-1}^{2}\right)^{i-1}\right]+\tilde{\alpha}_{1} \frac{h^{2}}{6}\left[\left(f_{m, n} s_{m, n}\right)^{i-1}\right. \\
& \left.-\left(f_{m, n-1} s_{m, n-1}\right)^{i-1}\right]+\tilde{p}_{n}
\end{aligned}
$$

$$
\begin{aligned}
& \tilde{p}_{n}=-(1-\theta)\left[\left(l_{m-1}, n s_{m-1}, n-v_{m-1}, f_{m-1, n}\right)-\left(l_{m-1}, n-1 s_{m-1}, n-1\right.\right. \\
& \left.\left.-v_{m-1, n-1} f_{m-1, n-1}\right)\right]+\frac{h}{2}\left[\tilde{\alpha}_{2}\left(f_{m-1, n}^{2}+f_{m-1, n-1}^{2}\right)+\tilde{\alpha}_{3}\left(f_{m-2, n}^{2}+f_{m-2, n-1}^{2}\right)-2 \beta\right] \\
& -\frac{h^{2}}{6}\left[\tilde{\alpha}_{2}\left(f_{m-1}, s_{m-1}, n-f_{m-1, n-1} s_{m-1, n-1}\right)\right. \\
& \left.+\tilde{\alpha}_{3}\left(f_{m-2}, n s_{m-2}, n-f_{m-2, n-1} s_{m-2, n-1}\right)\right]
\end{aligned}
$$

and ( ) denotes quantities that are evaluated outside of the main iteration loop.

\section{Shear Finite-Difference Equation}

For the shear equation, $g_{n}$ (eq. (16)) and $g_{n}^{\prime}$ (eq. (19)) are written as

$$
\begin{aligned}
& g_{n}=\theta f_{m, n}^{i}+(1-\theta) f_{m-1, n} \\
& g_{n}^{\prime}=\theta s_{m, n}^{i}+(1-\theta) s_{m-1, n}
\end{aligned}
$$




\section{APPENDIX A}

To obtain a linearized expression for $g_{n}^{\prime \prime}$, equation (22) is rewritten in the following form:

$$
\begin{aligned}
{\left[\theta\left(2 \ell_{m, n}^{i}-1\right)+(1-\theta)\left(2 l_{m-1, n}-1\right)\right] g_{n}^{\prime \prime}=} & \theta\left(v_{m, n} s_{m, n}\right)+(1-\theta)\left(v_{m-1, n} s_{m-1, n}\right) \\
& +\beta\left[\theta\left(f_{m, n}^{2}\right)^{i}+(1-\theta) f_{m-1, n}^{2}-1\right] \\
& +\xi\left[\tilde{c}_{1}\left(f_{m, n}^{2}\right)^{i}-\tilde{c}_{2} f_{m-1, n}^{2}+\tilde{c}_{3} f_{m-2, n}^{2}\right] \\
& -\theta t_{m, n}^{\prime}\left|s_{m, n}^{i-1}\right| s_{m, n}^{i-1} \\
& -(1-\theta) t_{m-1, n}^{\prime}\left|s_{m-1, n}\right| s_{m-1, n}
\end{aligned}
$$

Now define

$$
\begin{aligned}
& \tilde{\alpha}_{4}=\xi \tilde{c}_{1}+\theta \beta \\
& \tilde{\alpha}_{5}=-\xi \tilde{c}_{2}+(1-\theta) \beta \\
& \tilde{\alpha}_{6}=\xi \tilde{c}_{3}
\end{aligned}
$$

and

$\tilde{\phi}_{n}=(1-\theta)\left(v_{m-1, n} s_{m-1, n}-t_{m-1, n}^{\prime}\left|s_{m-1, n}\right| s_{m-1, n}\right)+\tilde{\alpha}_{5} f_{m-1, n}^{2}+\tilde{\alpha}_{6} f_{m-2, n}^{2}-\beta$ 
Equation (A30) can be rewritten as

$$
\begin{aligned}
& {\left[\theta\left(2 \ell_{m, n}^{i}-1\right)+(1-\theta)\left(2 \ell_{m-1, n}-1\right)\right] g_{n}^{n}=\theta\left(v_{m, n} s_{m, n}\right) i+\tilde{\alpha}_{4}\left(f_{m, n}^{2}\right)^{i}} \\
& -\theta t_{m, n}^{\prime}\left|s_{m, n}^{i-1}\right| s_{m, n}^{i-1}+\tilde{\phi}_{n}
\end{aligned}
$$

After linearizing equation (A35) via Newton's method, the following linearized expression is obtained for $g_{n}^{n}$ :

$$
\begin{aligned}
g_{n}^{\prime \prime}= & \left\{\theta\left[v_{m, n}^{i-1} s_{m, n}^{i}+v_{m, n}^{i} s_{m, n}^{i-1}-\left(v_{m, n} s_{m, n}\right)^{i-1}\right]+\tilde{\alpha}_{4}\left[2 f_{m, n}^{i-1} f_{m, n}^{i}-\left(f_{m, n}^{2}\right)^{i-1}\right]\right. \\
& \left.-\theta t_{m, n}^{\prime}\left|s_{m, n}^{i-1}\right| s_{m, n}^{i-1}+\tilde{\phi}_{n}-2 \theta\left[t_{m, n}\left|s_{m, n}^{i}\right|-\left(l_{m, n}-1\right)\right] s_{m-(1-\theta), n}^{\prime}\right\} H_{n}
\end{aligned}
$$

where

$$
H_{n}=\left[\theta\left(2 \ell_{m, n}^{i-1}-1\right)+(1-\theta)\left(2 \ell_{m-1, n}-1\right)\right]^{-1}
$$

Upon substitution of equations (A28), (A29), and (A36) into equation (11), the shear equation can be rewritten as

$a_{21} s_{m, n-1}^{i}+b_{21} s_{m, n}^{i}+a_{22} f_{m, n-1}^{i}+b_{22} f_{m, n}^{i}+a_{23} v_{m, n-1}^{i}+b_{23} v_{m, n}^{i}=q_{n}$

where

$$
a_{21}=-\frac{h}{2} \theta-\theta \frac{h^{2}}{12}\left\{v_{m, n-1}^{i-1}-2 t_{m, n-1}\left[s_{m-(1-\theta), n-1}^{\prime}\right]^{i-1} k_{n-1}\right\}
$$




\section{APPENDIX A}

$$
\begin{aligned}
& b_{21}=-\frac{h}{2} \theta+\theta \frac{h^{2}}{12}\left\{v_{m, n}^{i-1}-2 t_{m, n}\left[s_{m}^{\prime}-(1-\theta), n\right]^{i-1} k_{n}\right\} \\
& a_{22}=-\theta+\tilde{\alpha}_{4} \frac{h^{2}}{6} H_{n-1} f_{m, n-1}^{i-1} \\
& b_{22}=\theta+\tilde{\alpha}_{4} \frac{h^{2}}{6} H_{n} \mathrm{f}_{m, n}^{i-1} \\
& a_{23}=-\theta \frac{h^{2}}{12} H_{n-1} s_{m, n-1}^{i-1} . \\
& b_{23}=\theta \frac{h^{2}}{12} H_{n} s_{m, n}^{i-1} \\
& q_{n}=-\frac{h^{2}}{12}\left(H _ { n } \left\{-\theta\left(v_{m, n} s_{m, n}\right)^{i-1}-\tilde{\alpha}_{4}\left(f_{m, n}^{2}\right)^{i-1}-\theta t_{m, n}^{\prime}\left|s_{m, n}^{i-1}\right| s_{m, n}^{i-1}\right.\right. \\
& \left.+\tilde{\phi}_{n}+2 \theta\left(e_{m, n}^{i-1}-1\right)\left[s_{m}^{i}-(1-\theta), n\right]^{i-1}\right\}-H_{n-1}\left\{-\theta\left(v_{m, n-1} s_{m, n-1}\right)^{i-1}\right. \\
& -\tilde{\alpha}_{4}\left(f_{m, n-1}^{2}\right)^{i-1}-\theta t_{m, n-1}^{\prime}\left|s_{m, n-1}^{i-1}\right| s_{m, n-1}^{i-1}+\tilde{\phi}_{n-1} \\
& \left.\left.+2 \theta\left(e_{m, n-1}^{i-1}-1\right)\left[s_{m}^{\prime}-(1-\theta), n-1\right]^{i-1}\right\}\right)+\tilde{q}_{n} \\
& \tilde{q}_{n}=-(1-\theta)\left[f_{m-1, n}-f_{m-1, n-1}-\frac{h}{2}\left(s_{m-1, n}+s_{m-1, n-1}\right)\right]
\end{aligned}
$$

with $K_{n}= \pm 1$, having the same sign as $s_{m, n}^{i-1}$. 


\section{Continuity Finite-Difference Equation}

Equations (17), (20), and (23) can be written as

$g_{n}=\theta v_{m, n}^{i}+(1-\theta) v_{m-1, n}$

$g_{n}^{\prime}=-2 \xi\left(\tilde{c}_{1} f_{m, n}^{i}-\tilde{c}_{2} f_{m-1, n}+\tilde{c}_{3} f_{m-2, n}\right)-\theta f_{m, n}^{i}-(1-\theta) f_{m-1, n}$

$g_{n}^{\prime \prime}=-2 \xi\left(\tilde{c}_{1} s_{m, n}^{i}-\tilde{c}_{2} s_{m-1, n}+\tilde{c}_{3} s_{m-2, n}\right)-\theta s_{m, n}^{i}-(1-\theta) s_{m-1, n}$

Define

$$
\begin{aligned}
& \tilde{\alpha}_{7}=-2 \xi c_{1}-\theta \\
& \tilde{\alpha}_{8}=2 \xi c_{2}-(1-\theta) \\
& \tilde{\alpha}_{9}=-2 \xi c_{3}
\end{aligned}
$$

Then, substitution of equations (A47), (A48), and (A49) into equation (11) yields the following equation:

$\theta\left(v_{m, n}^{i}-v_{m, n-1}^{i}\right)+(1-\theta)\left(v_{m-1, n}-v_{m-1, n-1}\right)-\frac{h}{2}\left[\widetilde{\alpha}_{7}\left(f_{m, n}^{i}+f_{m, n-1}^{i}\right)\right.$

$\left.+\tilde{\alpha}_{8}\left(f_{m-1, n}+f_{m-1, n-1}\right)+\tilde{\alpha}_{9}\left(f_{m-2, n}+f_{m-2, n-1}\right)\right]+\frac{h^{2}}{12}\left[\tilde{\alpha}_{7}\left(s_{m, n}^{i}-s_{m, n-1}^{i}\right)\right.$

$\left.+\tilde{\alpha}_{8}\left(s_{m-1, n}-s_{m-1, n-1}\right)+\tilde{\alpha}_{9}\left(s_{m-2, n}-s_{m-2, n-1}\right)\right]=0$

which can be written as

$a_{31} s_{m, n-1}^{i}+b_{31} s_{m, n}^{i}+a_{32} f_{m, n-1}^{i}+b_{32} f_{m, n}^{i}+a_{33} v_{m, n-1}^{i}+b_{33} v_{m, n}^{i}=r_{n}$ 
where

$$
\begin{aligned}
& a_{31}=-\tilde{\alpha}_{7} \frac{h^{2}}{12} \\
& b_{31}=\tilde{\alpha}_{7} \frac{h^{2}}{12} \\
& a_{32}=-\tilde{\alpha}_{7} \frac{h}{2} \\
& b_{32}=-\tilde{\alpha}_{7} \frac{h}{2} \\
& a_{33}=-\theta \\
& b_{33}=\theta \\
&\left.+\tilde{\alpha}_{9}\left(f_{m-2, n}+f_{m-2, n}\right)\right]-\frac{h^{2}}{12}\left[\tilde{\alpha}_{8}\left(s_{m-1, n}-s_{m-1}, n-1\right)\right. \\
& r_{n}= \tilde{r}_{n} \\
& \tilde{r}_{n}=-(1-\theta)\left(v_{m-1, n}-v_{m-1}, n-1\right)+\frac{h}{2}\left[\tilde{\alpha}_{8}\left(f_{m-1}, n+f_{m-1, n-1}\right)\right.
\end{aligned}
$$




\section{APPENDIX B}

\section{METHOD OF SOLUTION (B4S) OF FINITE-DIFFERENCE EQUATIONS}

The linear system of finite-difference equations (24) can be solved in the relatively simple manner which is presented in this appendix. If equations (24) are applied at $n=2 \quad(n=1$ being the surface and $n=N$ locating the outer boundary) and the boundary conditions $f_{1}=v_{1}=0$ are applied, three equations with four unknowns are obtained. Thus, three of the unknowns may be expressed in terms of the fourth unknown.

Since $f_{N}$ is known at the outer boundary, it is convenient to solve for $s_{1}$, $s_{2}$, and $v_{2}$ in terms of $f_{2}$, in anticipation of the general form of the recursion relations to be derived subsequently. Thus, at $n=2$, equations (24) can be rewritten as

$$
\left(s_{2}, s_{1}, v_{2}\right)^{T}=c^{-1}\left[\vec{w}_{2}-\left(b_{12}, b_{22}, b_{32}\right)^{T_{f_{2}}}\right]
$$

where

$$
c=\left[\begin{array}{lll}
b_{11} & a_{11} & b_{13} \\
b_{21} & a_{21} & b_{23} \\
b_{31} & a_{31} & b_{33}
\end{array}\right]
$$

Next by applying equations (24) at $n=3$ and using equation (BI) to eliminate $s_{2}$ and $v_{2}$ as unknowns, the result is again three equations and four unknowns, namely, $f_{2}, f_{3}, v_{3}$, and $s_{3}$. Thus, three of the unknowns may again be expressed in terms of the fourth unknown; that is, solve for $f_{2}, v_{3}$, and $s_{3}$, in terms of $f_{3}$ (this order is dictated by the choice at $n=2$ which was made with the consideration of the outer boundary condition $f_{N}=1$ ). If this procedure is repeated for $n=4, \ldots . ., N$, the following general form is obtained for the three unknowns written in terms of the fourth:

$$
\left.\begin{array}{l}
s_{n}=d_{n}^{(1)}+e_{n}^{(1)} f_{n} \\
f_{n-1}=d_{n}^{(2)}+e_{n}^{(2)} f_{n} \\
v_{n}=d_{n}^{(3)}+e_{n}^{(3)} f_{n}
\end{array}\right\}
$$

where the superscripts 1,2, and 3 in parentheses identify the coefficients associated with the unknowns $s, f$, and $v$, respectively. 
The coefficients $d_{n}^{(1)}, a_{n}^{(2)}, d_{n}^{(3)}, e_{n}^{(1)}, e_{n}^{(2)}$, and $e_{n}^{(3)}$ are given

$$
\left[d_{n}^{(1)}, a_{n}^{(2)}, d_{n}^{(3)}\right]^{T}=D_{n}^{-1}\left[\left(p_{n}, g_{n}, r_{n}\right)^{T}-\left(a_{11}, a_{21}, a_{31}\right)^{T} d_{n-1}^{(1)}-\left(a_{13}, a_{23}, a_{33}\right)^{T} d_{n-1}^{(3)}\right]
$$

$\left[e_{n}^{(1)}, e_{n}^{(2)}, e_{n}^{(3)}\right]^{T}=-D_{n}{ }^{-1}\left(b_{12}, b_{22}, b_{32}\right)^{T}$

with

$$
D_{n}=\left[\begin{array}{lll}
b_{11} & a_{12}+a_{11} e_{n-1}^{(1)}+a_{13} e_{n-1}^{(3)} & b_{13} \\
b_{21} & a_{22}+a_{21} e_{n-1}^{(1)}+a_{23} e_{n-1}^{(3)} & b_{23} \\
b_{31} & a_{32}+a_{31} e_{n-1}^{(1)}+a_{33} e_{n-1}^{(3)} & b_{33}
\end{array}\right]
$$

Examination of equations (B3) to (B8) reveals that although the solution for the dependent variables $s_{n}, f_{n}$, and $v_{n}$ cannot be computed until the outer boundary is reached where $f_{N}=1$, the coefficients $d_{n}^{(1)}, d_{n}^{(2)}, a_{n}^{(3)}, e_{n}^{(1)}$, $e_{n}^{(2)}$, and $e_{n}^{(3)}$ can be computed if the initial values $d_{2}^{(1)}, d_{2}^{(3)}, e_{2}^{(1)}$, and $e_{2}^{(3)}$ are known. These initial values are found by comparing equations (B3) and (B5) evaluated at $n=2$ with equations (B1). The initial values are

$$
\begin{aligned}
& {\left[a_{2}^{(1)}, \psi, a_{2}^{(3)}\right]^{T}=c^{-1}\left(p_{2}, q_{2}, r_{2}\right)^{T}} \\
& {\left[e_{2}^{(1)}, \omega, e_{2}^{(3)}\right]^{T}=-c^{-1}\left(b_{12}, b_{22}, b_{32}\right)^{T}}
\end{aligned}
$$


where $\psi$ and $\omega$ are coefficients to compute $s_{1}$ from

$$
s_{1}=\psi+\omega f_{2}
$$

When the outer boundary is reached, equations (B3) to (B5) can be solved for $s_{N}, f_{N-1}$, and $v_{N}$ since the outer boundary condition $f_{N}=l$ can be applied. Next $s_{\mathrm{N}-1}, f_{\mathrm{N}-2}$, and $v_{\mathrm{N}-1}$ can be computed since $f_{\mathrm{N}-1}$ is known; and likewise all the values of $s_{n}, f_{n}$, and $v_{n}$ proceeding from the outer boundary toward the solid boundary can be computed. 


\section{REFERENCES}

1. Keller, Herbert B.: Numerical Methods for Two-Point Boundary-Value Problems. Blaisdell Pub. Co., c.1968.

2. Orszag, Steven A.; and Israeli, Moshe: Numerical Simulation of Viscous Incompressible Flows. Annual Review of Fluid Mechanics, Volume 6, Milton Van Dyke, Walter G. Vincenti, and J. V. Wehausen, eds., Annu. Rev., Inc., 1974, pp. 281-318.

3. Hirsh, Richard S.: Higher Order Accurate Difference Solutions of Fluid Mechanics Problems by a Compact Differencing Technique. J. Comput. Phys., vol. 19, no. 1, Sept. 1975, pp. 90-109.

4. Krause, E.; Hirschel, E. H.; and Kordulla, W.: Fourth Order "Mehrstellen"Integration for Three-Dimensional Turbulent Boundary Layers. AIAA Computational Fluid Dynamics Conference, July 1973, pp. 92-102.

5. Peters, Norbert: Boundary Layer Calculation by a Hermitian Finite Difference Method. Proceedings of the Fourth International Conference on Numerical Methods in Fluid Mechanics, Volume 35 of Lecture Notes in Physics, Robert D. Richtmyer, ed., Springer-Verlag, 1975, pp. 313-318.

6. Rubin, Stanley G.; and Graves, Randolph A., Jr.: Viscous Flow Solutions With a Cubic Spline Approximation. Comput. \& Fluids, vol. 3, no. 1, Mar. 1975, pp. 1-36. See also NASA TR R-436, 1975.

7. Rubin, Stanley G.; and Graves, Randolph A., Jr.: A Cubic Spline Approximation for Problems in Fluid Mechanics. NASA TR R-436, 1975.

8. Rubin, S. G.; and Khosla, P. K.: Higher-Order Numerical Solutions Using Cubic Splines. AIAA 2nd Computational Fluid Dynamics Conference, June 1975, pp. 55-66. (Also available as NASA CR-2653, 1976.)

9. Rubin, S. G.; and Khosla, P. K.: Higher-Order Numerical Methods Derived From Three-Point Polynomial Interpolation. NASA CR-2735, 1976.

10. Adam, Yves: A Hermitian Finite Difference Method for the Solution of Parabolic Equations. Comput. \& Math. With Appl., vol. 1, no. 3/4, 1975, pp. 393-406.

11. Adam, Yves: Highly Accurate Compact Implicit Methods and Boundary Conditions. J. Comput. Phys., vol. 24, no. 1, May 1977, pp. 10-22.

12. Blottner, F. G.: Investigation of Some Finite-Difference Techniques for Solving the Boundary Layer Equations. Comput. Methods Appl. Mech. \& Eng., vol. 6, no. 1, 1975, pp. 1-30.

13. Keller, Herbert B.: A New Difference Scheme for Parabolic Problems. Numerical Solution of Partial Differential Equations - II, Bert Hubbard, ed., Academic Press, Inc., 1971, pp. 327-350. 
14. White, Andrew Benjamin, Jr.: Numerical Solution of Two-Point BoundaryValue Problems. Ph. D. Thesis, California Inst. Technol., 1974.

15. Keller, H. B.: Numerical Solution of Boundary Value Problems for Ordinary Differential Equations: Survey and Some Recent Results on Difference Methods. Numerical Solutions of Boundary Value Problems for Ordinary Differential Equations, A. K. Aziz, ed., Academic Press, Inc., 1975, pp. 27-88.

16. Schlichting, Hermann (J. Kestin, transl.): Boundary-Layer Theory. Sixth ed. McGraw-Hill Book Co., Inc., C.1968.

17. Görtler, Henry: A New Series for the Calculation of Steady Laminar Boundary Layer Flows. J. Math. \& Mech., vol. 6, no. 1, Jan. 1957, pp. 1-66.

18. Michel, R.; Quémard, C.; and Durant, R.: Hypotheses on the Mixing Length and Application to the Calculation of the Turbulent Boundary Layers. Computation of Turbulent Boundary Layers - 1968 AFOSR-IFP-Stanford Conference, Volume 1, S. J. Kline, M. V. Morkovin, G. Sovran, and D. J. Cockrell, eds., Stanford Univ., c.1969, pp. 195-212A.

19. Liniger, Werner; and Willoughby, Ralph A.: Efficient Integration Methods for Stiff Systems of Ordinary Differential Equations. SIAM J. Numer. Anal., vol. 7, no. 1, Mar. 1970, pp. 47-66.

20. Cebeci, Tuncer; and Smith, A. M. O.: Analys is of Turbulent Boundary Layers. Academic Press, Inc., 1974.

21. Rubin, S. G.; and Khosla, P. K.: An Integral Method for Boundary Layer Equations. POLY M/AE Rep. No. 77-12 (Grant No. AFOSR 74-2635, Polytech. Inst. New York, July 1977. (Available from DDC as AD A044 571.)

22. Keller, Herbert B.; and Cebeci, Tuncer: Accurate Numerical Methods for Boundary-Layer Flows. II: Two-Dimensional Turbulent Flows. AIAA J., vol. 10, no. 9, Sept. 1972, pp. 1193-1199.

23. Pereyra, Victor: High Order Finite Difference Solution of Differential Equations. Tech. Rep. STAN-CS-73-348 (Contract NSF-GJ-35135), Dep. Comput. Sci., Stanford Univ., Apr. 1973. (Available from NTIS as PB-222 859.)

24. Blottner, F. G.: Variable Grid Scheme Applied to Turbulent Boundary Layers. Comput. Methods Appl. Mech. \& Eng., vol. 4, no. 2, Sept. 1974, pp. 179-194.

25. Wornom, Stephen F.: A Critical Study of Higher-Order Numerical Methods for Solving the Boundary-Layer Equations. AIAA 3rd Computational Fluid Dynamics Conference, June 1977, pp. 61-71. (Available as AIAA Paper No. 77-637.) 


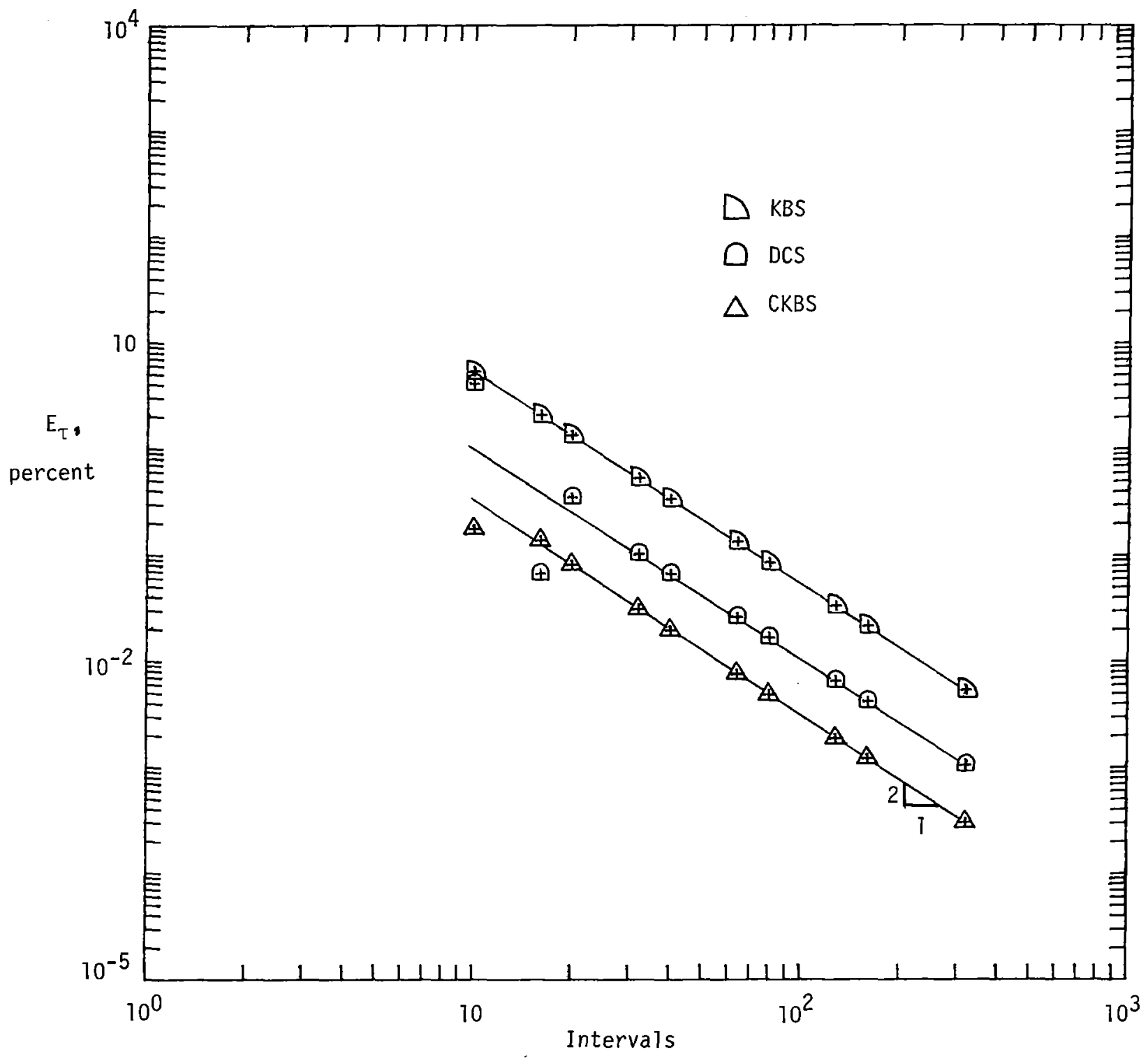

Figure 1.- Accuracy of wall shear with second-order methods. Laminar flow; $B=0 ; s_{1}=0.469599988$ (exact). 


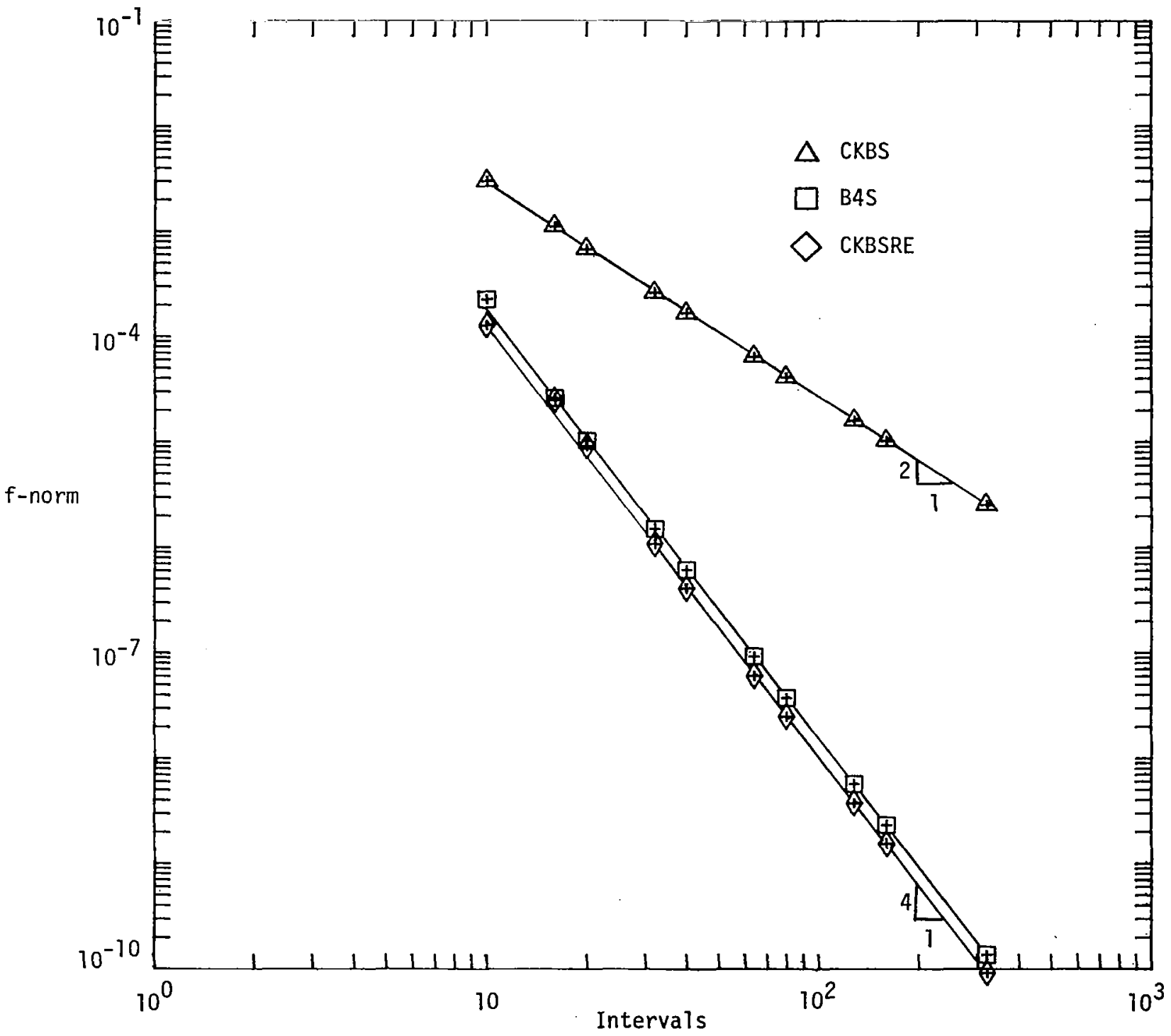

(a) CKBSRE with B4S.

Figure 2.- Comparison of higher order methods for laminar flow. $\beta=1 ; s_{1}=1.232587657$ (exact). 


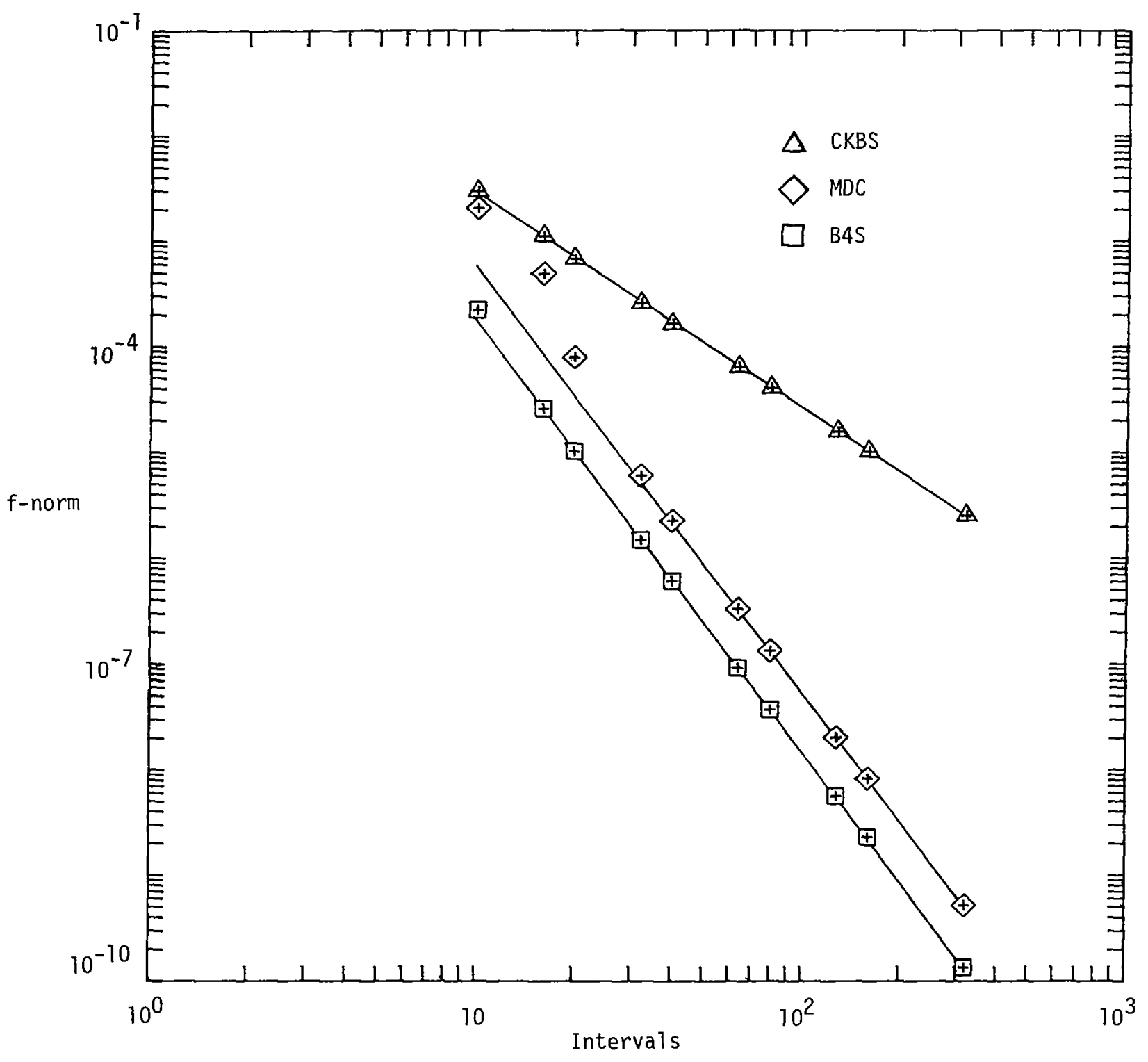

(b) MDC with B4S.

Figure 2.- Continued. 


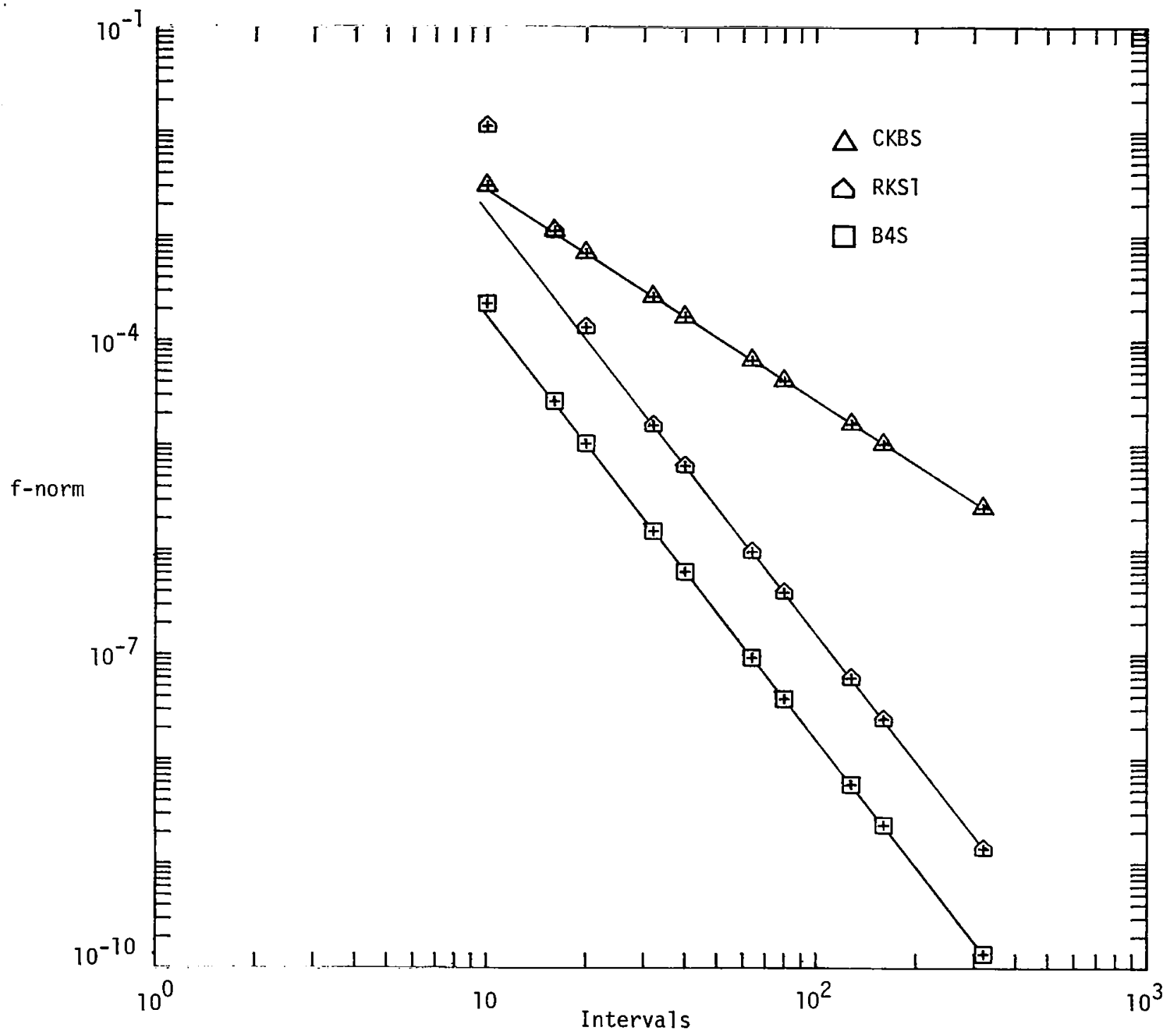

(c) RKS1 with B4S.

Figure 2.- Continued. 


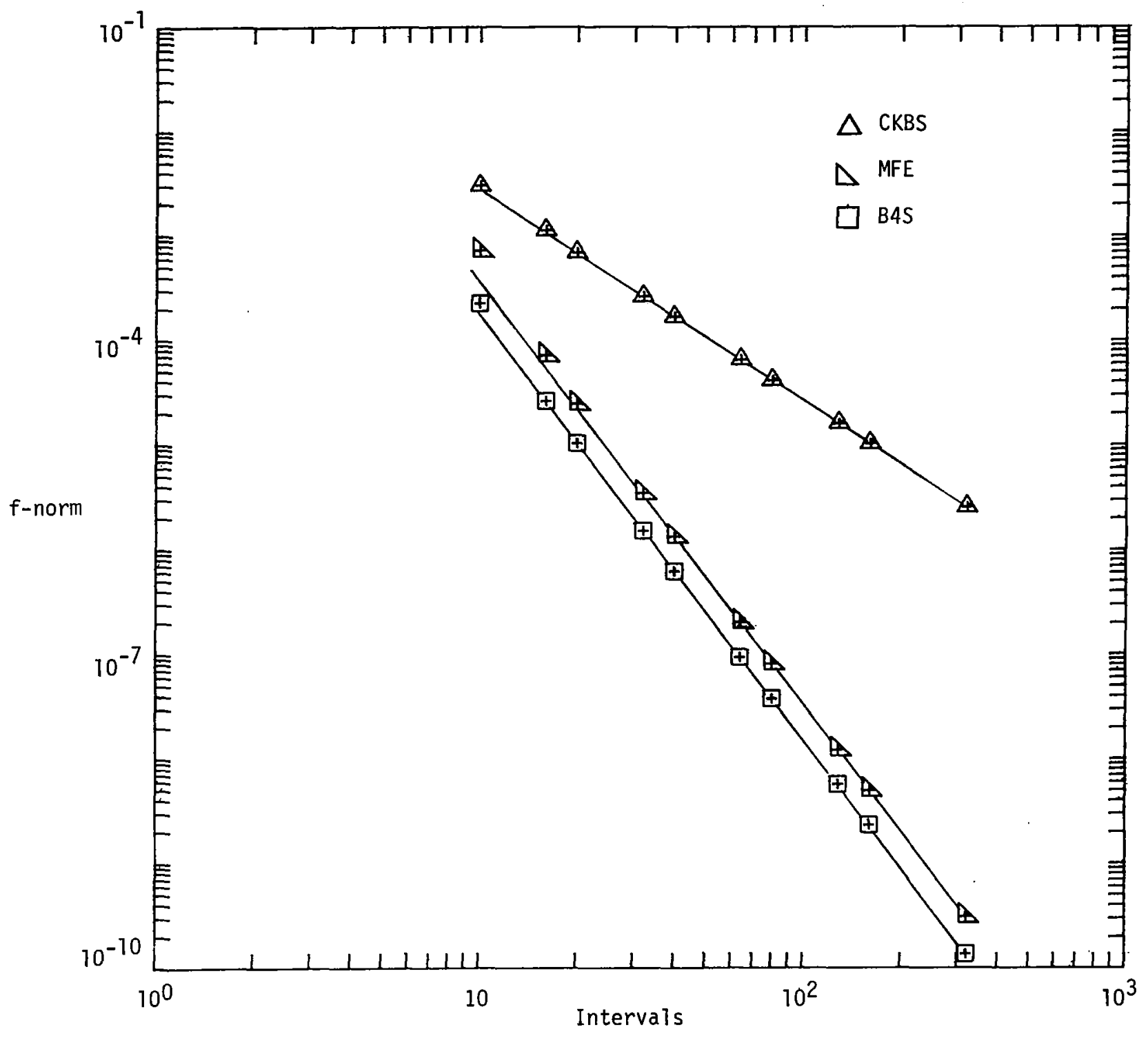

(d) MFE with B4S.

Figure 2.- Concluded. 


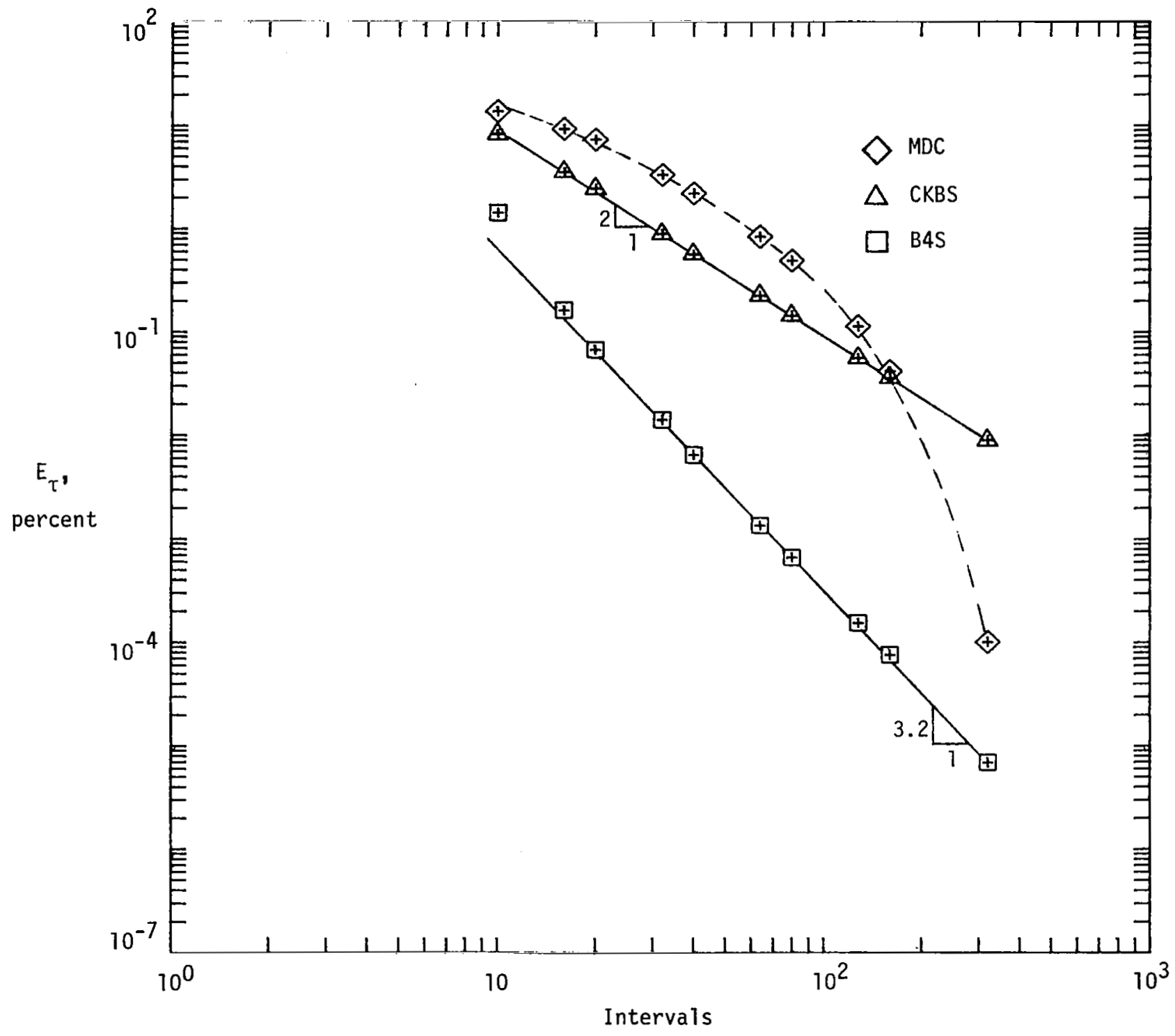

(a) MDC with B4S,

Figure 3.- Comparison of higher order methods for a model turbulent-flow problem with $B=0 . R_{L}=1.88 \times 10^{6} ; \quad C_{f} \boxminus 2.834124 \times 10^{-3}$ (exact). 


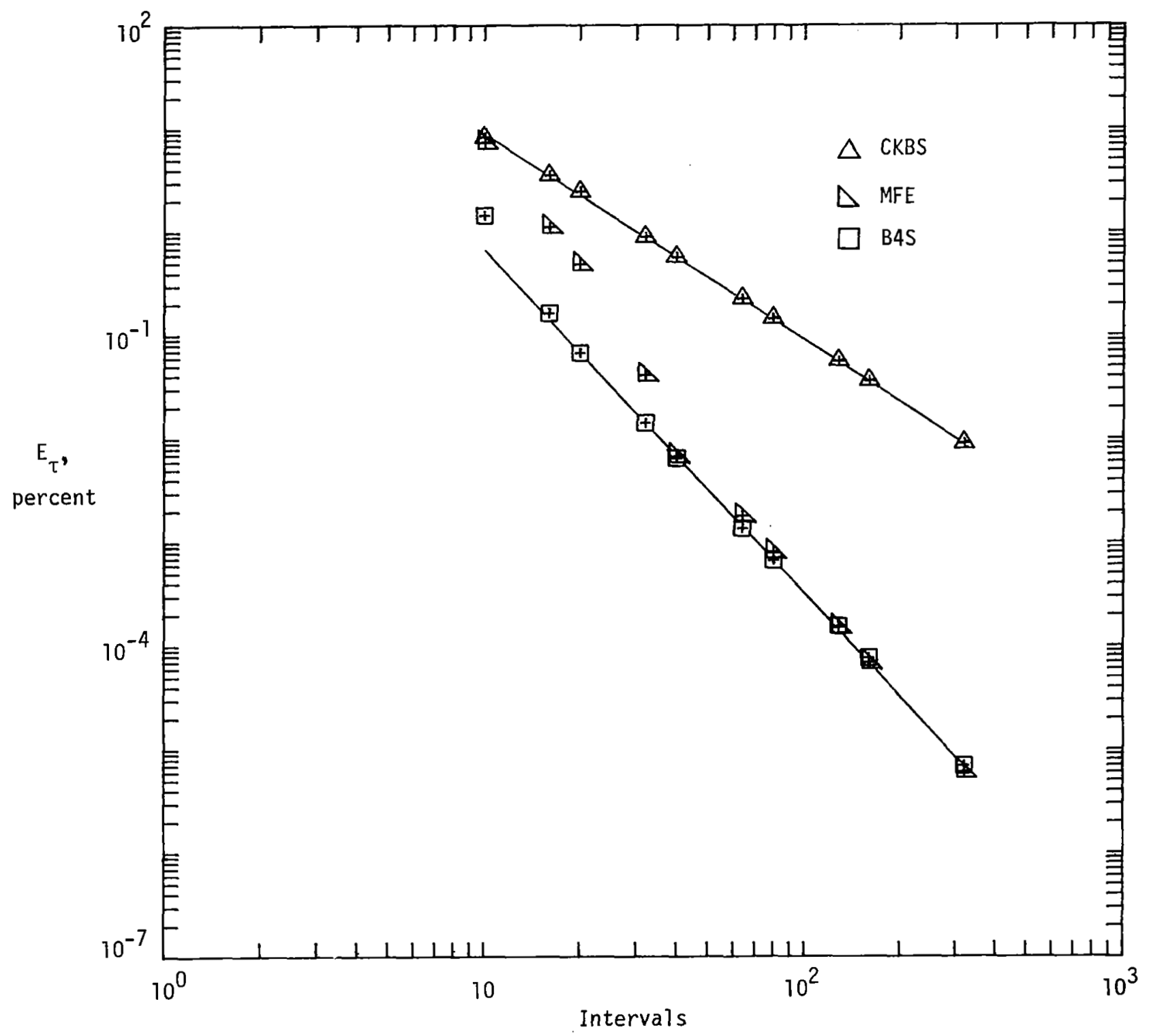

(b) MFE with B4S.

Figure 3.- Continued. 


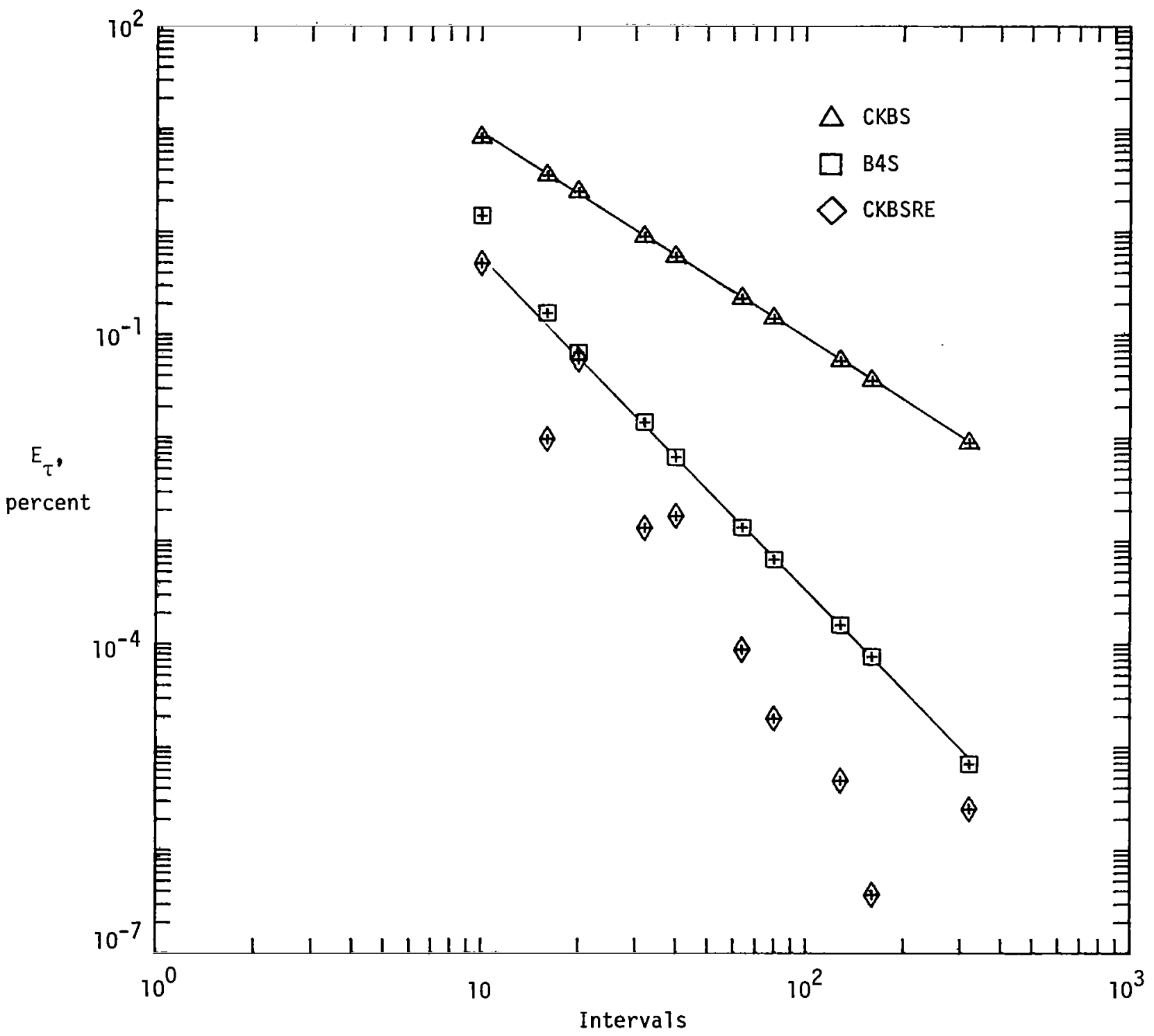

(c) CKBSRE with B4S.

Figure 3.- Continued. 


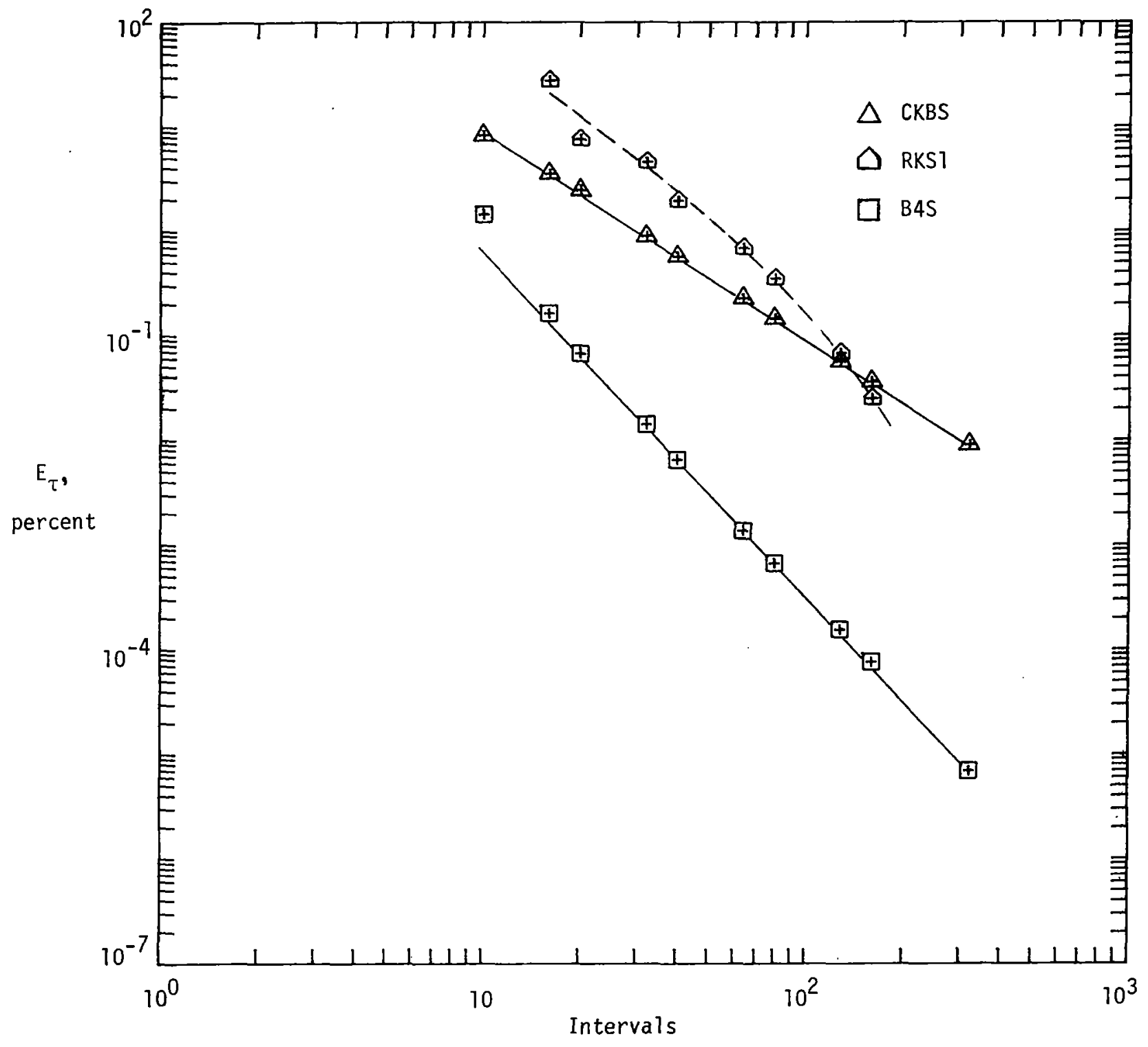

(d) RKS1 with B4S.

Figure 3.- Concluded. 


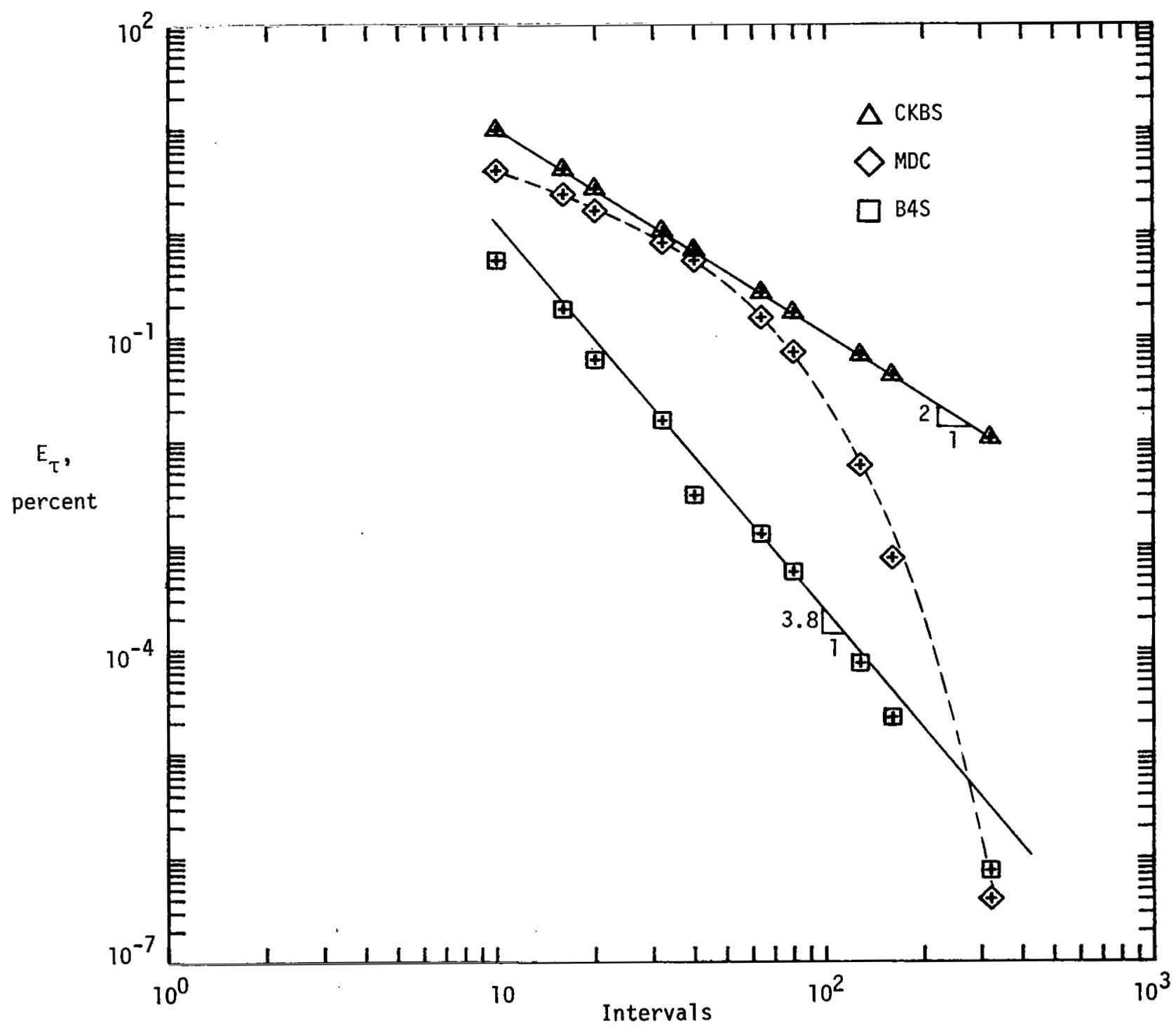

(a) MDC with B4S.

Figure 4.- Comparison of higher order methods for model turbulent-flow problem with $B=0.5 . \quad R_{L}=1.88 \times 10^{6} ; \quad C_{f}=3.530597 \times 10^{-3}$ (exact). 


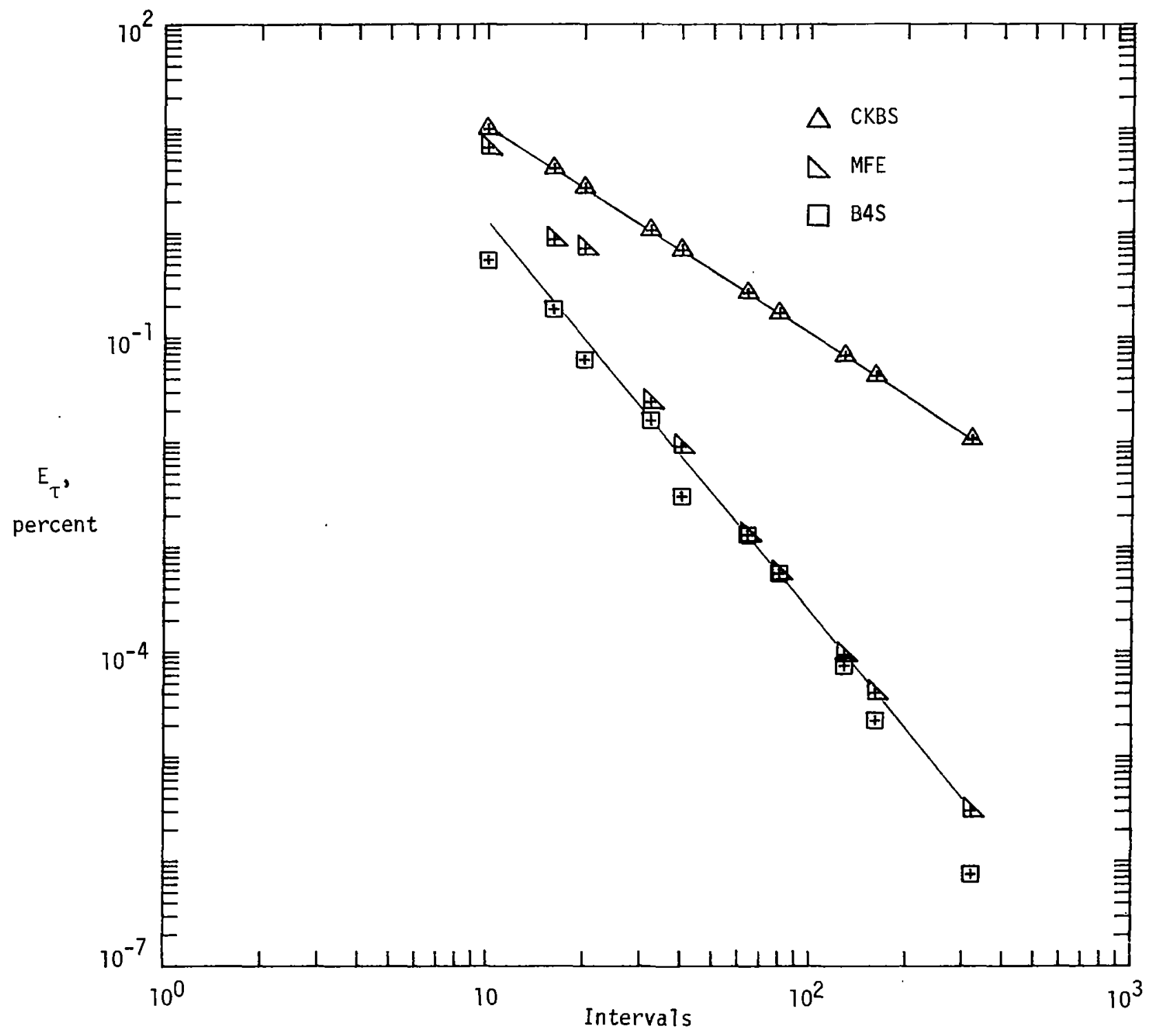

(b) MFE with B4S.

Figure 4.- Continued. 


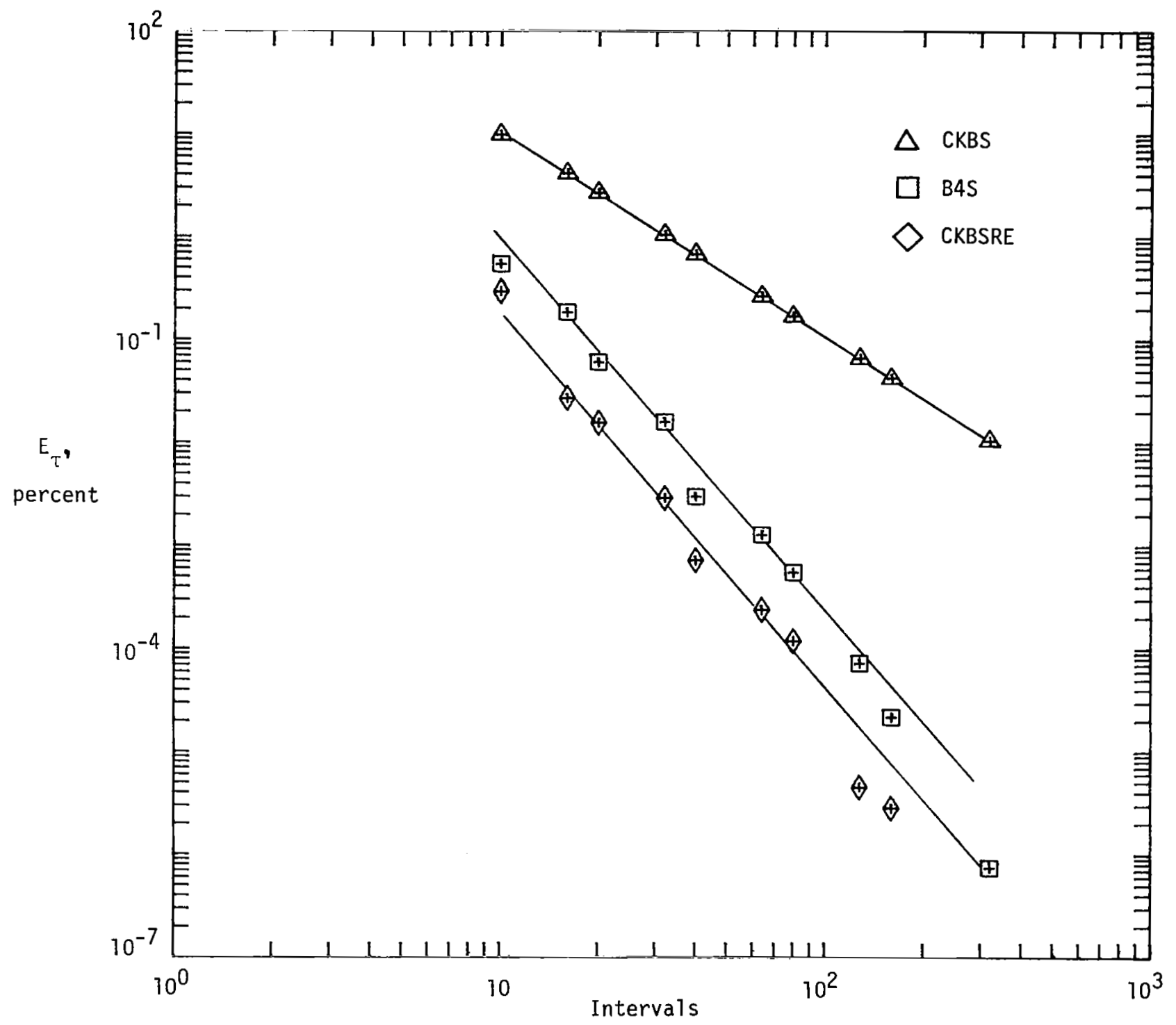

(c) CKBSRE with B4S.

Figure 4.- Concluded. 


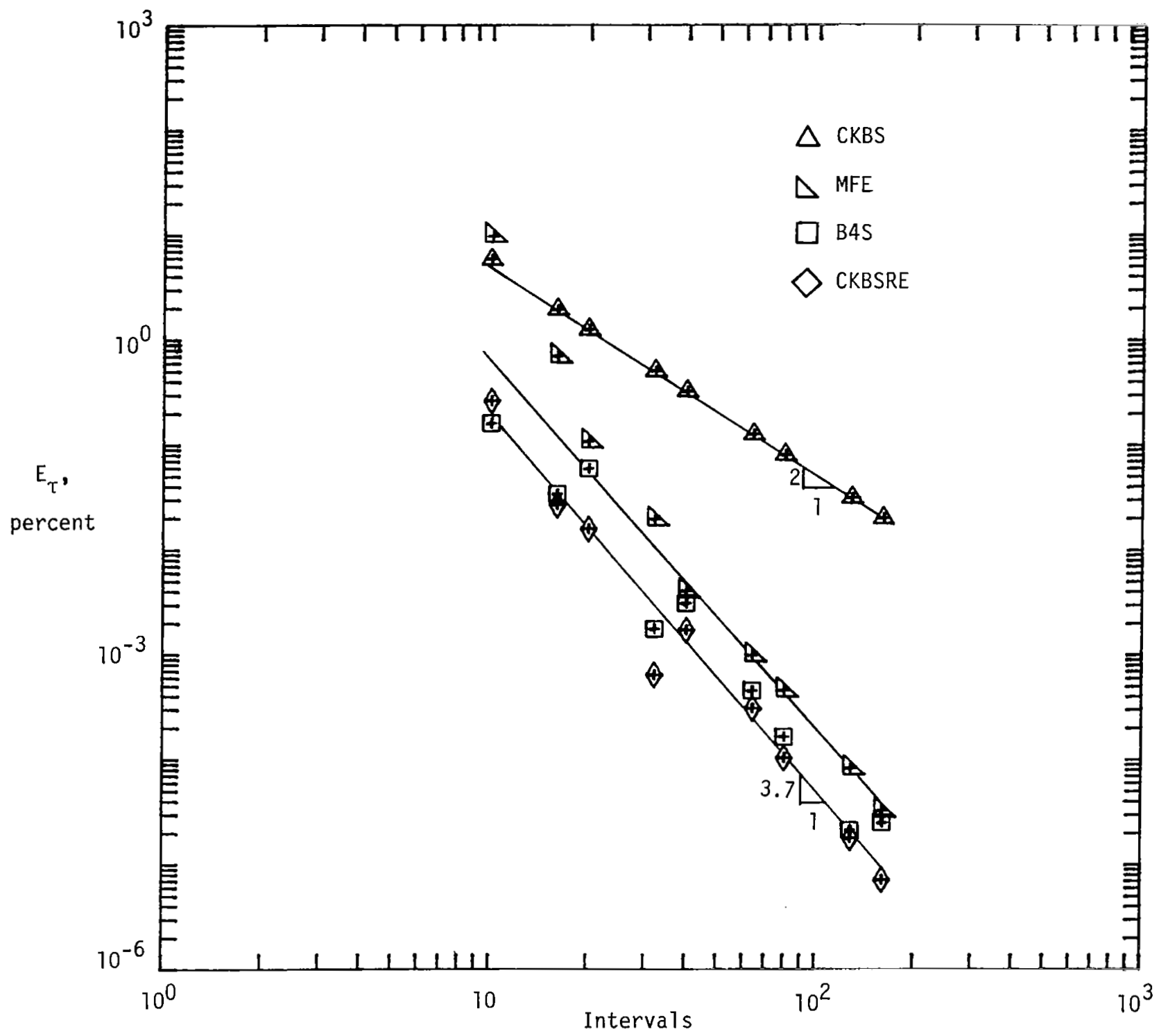

Figure 5.- Accuracy of wall shear for nonsimilar turbulent flow. $B$ घ 0 ; $\xi=1.88 ; \mathrm{R}_{\mathrm{X}}$ = $1.88 \times 10^{6} ; \mathrm{C}_{\mathrm{f}}=3.001974 \times 10^{-3}$ (exact). 


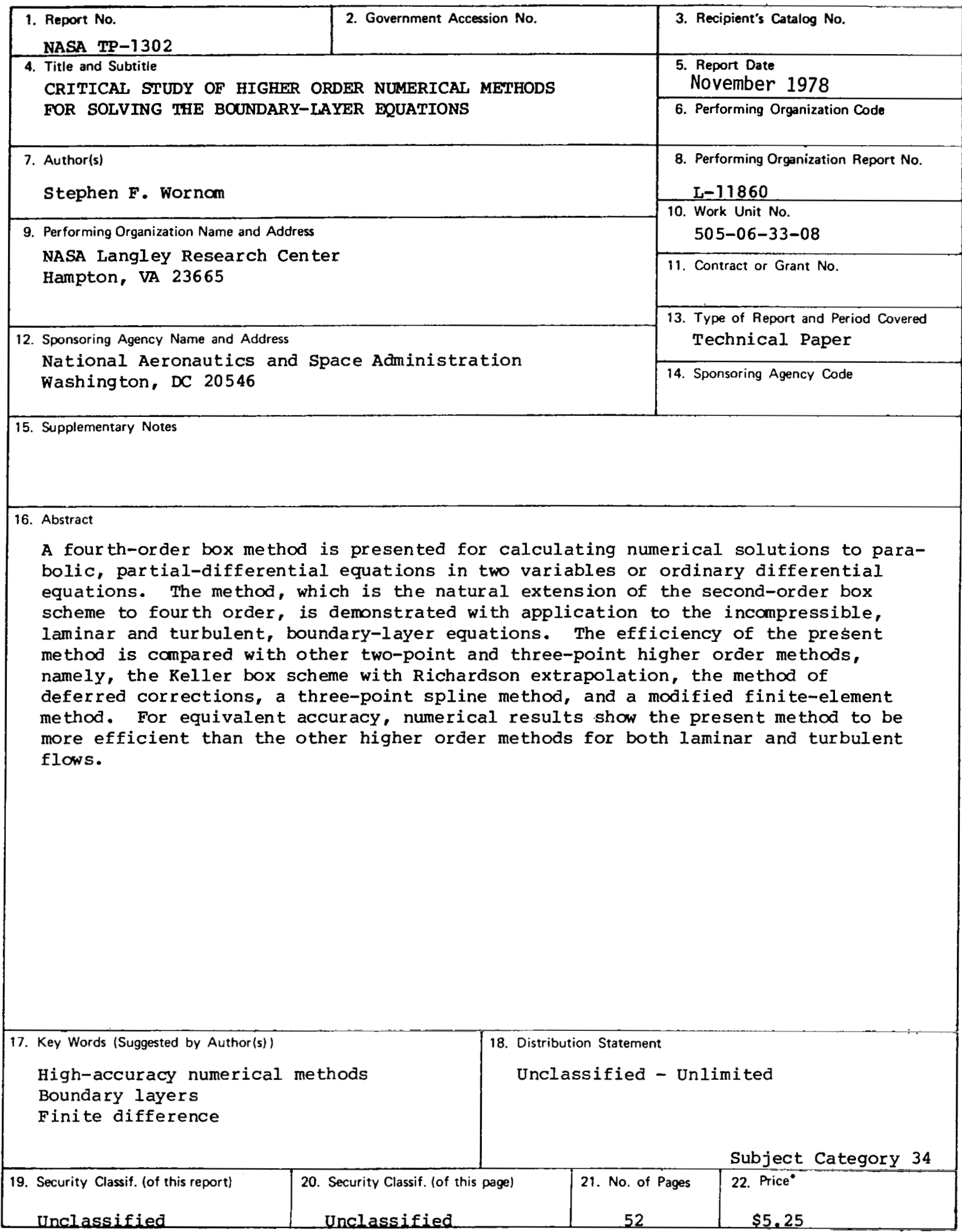

*For sale by the National Technical Information Service, Springfield, Virginia 22161 
National Aeronautics and

THIRD-CLASS BULK RATE Space Administration

Washington, D.C.

20546

Official Business

Penalty for Private Use, $\$ 300$.

$1110, A$,

102078 S00903DS

DEPT OF THE AIR FORCE

AF WEAPONS LAEORATORY

ATTN : TECHNICAL IIERARY (SUL)

RIRTLAND AFB NM 87117
Postage and Fees Paid

National Aeronautics and

Space Administration

NASA-451

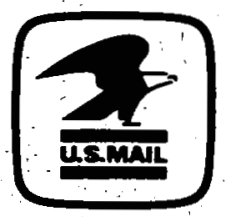

